\title{
Could the clinical interpretability of subgroups detected using clustering methods be improved by using a novel two-stage approach?
}

Peter Kent ${ }^{1 *}$, Mette Jensen Stochkendahl ${ }^{2}$, Henrik Wulff Christensen ${ }^{2}$ and Alice Kongsted ${ }^{1,2}$

\begin{abstract}
Background: Recognition of homogeneous subgroups of patients can usefully improve prediction of their outcomes and the targeting of treatment. There are a number of research approaches that have been used to recognise homogeneity in such subgroups and to test their implications. One approach is to use statistical clustering techniques, such as Cluster Analysis or Latent Class Analysis, to detect latent relationships between patient characteristics.

Influential patient characteristics can come from diverse domains of health, such as pain, activity limitation, physical impairment, social role participation, psychological factors, biomarkers and imaging. However, such 'whole person' research may result in data-driven subgroups that are complex, difficult to interpret and challenging to recognise clinically.

This paper describes a novel approach to applying statistical clustering techniques that may improve the clinical interpretability of derived subgroups and reduce sample size requirements.

Methods: This approach involves clustering in two sequential stages. The first stage involves clustering within health domains and therefore requires creating as many clustering models as there are health domains in the available data. This first stage produces scoring patterns within each domain. The second stage involves clustering using the scoring patterns from each health domain (from the first stage) to identify subgroups across all domains. We illustrate this using chest pain data from the baseline presentation of 580 patients.

Results: The new two-stage clustering resulted in two subgroups that approximated the classic textbook descriptions of musculoskeletal chest pain and atypical angina chest pain. The traditional single-stage clustering resulted in five clusters that were also clinically recognisable but displayed less distinct differences.

Conclusions: In this paper, a new approach to using clustering techniques to identify clinically useful subgroups of patients is suggested. Research designs, statistical methods and outcome metrics suitable for performing that testing are also described. This approach has potential benefits but requires broad testing, in multiple patient samples, to determine its clinical value. The usefulness of the approach is likely to be context-specific, depending on the characteristics of the available data and the research question being asked of it.
\end{abstract}

Keywords: Stratified health care, Clustering, Latent class analysis, Subgroups, Chest pain, Low back pain

\footnotetext{
* Correspondence: pkent@health.sdu.dk

${ }^{1}$ Department of Sports Science and Clinical Biomechanics, University of

Southern Denmark, Campusvej 55, Odense M 5230, Denmark

Full list of author information is available at the end of the article
} 


\section{Background}

There is increasing interest in stratified health care that targets treatments to individuals or homogenous subgroups of patients. The potential benefits of stratified health care are better treatment effects and reduced harm through a more precise matching of therapy to individual patients, and improved health system efficiency through more appropriate resource allocation [1]. This is especially the case in health conditions with high diagnostic and therapeutic uncertainty, where randomised controlled trials of a 'one size fits all' treatment approach have yielded disappointing effect sizes [2].

It is usual for patients to vary in their outcomes, due to prognostic diversity and differences in their treatment response, even when their condition may appear to be similar at the initial consultation. Using knowledge of the predictability of that diversity, stratified health care attempts to use baseline information about likely prognosis and likely treatment response to assist in the tailoring of treatment decisions [1]. Under that model, clinical decisions are based on predictions of likely outcomes. In contrast, there are other models of care that use the patient's actual response to treatment and initial outcomes to tailor subsequent treatment decisions, such as stepped care and adaptive treatment $[3,4]$.

Stratified health care requires prognostic models with sufficient predictive accuracy to be clinically useful. Such predictive accuracy either comes from an in-depth, although often elusive, understanding of what determines the variability in the outcomes of individual patients or more commonly, from recognising a sufficient amount of homogeneity in subgroups of patients that can usefully improve prediction of their outcomes [5].

There are a number of approaches that have been used to recognise homogeneity in subgroups of patients and to test its implications. The classic data-driven approach is to work backwards from an outcome in longitudinal data, such as people classified as responders or nonresponders to a treatment regimen. As these techniques use the outcome to determine the derived subgroups, statisticians refer to these approaches as 'supervised' techniques [6, 7]. Such supervised statistical techniques include: regression analysis, discriminant function analysis, recursive partitioning analysis, and classification and regression trees. An example of a study using regression analysis to create a clinical prognostic tool is that performed by Schellingerhout et al. 2010 [8], who created a score chart to estimate the probability of non-recovery at 6-month follow-up in patients with non-specific neck pain. The other main approach used in data-driven subgrouping is called 'unsupervised' [6] [7] because instead of working backwards from an outcome, these statistical techniques look for latent relationships between characteristics in cross-sectional data, usually baseline data.
The main limitation of supervised techniques is that subgroup formation is usually based on the prediction of one single outcome that may not be a comprehensive measure of the behaviour of the condition (for example, predictors of return-to-work may not be the same as predictors of recovery from pain). In contrast, unsupervised subgroup formation is based on baseline data only, and such subgroups are not dependent on one outcome or the efficacy of current treatments. Therefore, an advantage of this approach is that subgroups detected in this way can later be studied against a range of treatments and outcomes. The major disadvantage of this method is that because subgroups are not modelled using a clinical outcome, subgroups derived using unsupervised techniques may have no clinical relevance [9]. Therefore, such subgroups need to be rigorously tested for predictive validity. Two examples of studies that used unsupervised clustering techniques to derive subgroups from baseline psychological data and then investigate their predictive validity in longitudinal data are those conducted by Beneciuk et al. 2012 [10] and Westman et al. 2011 [11].

There is an increasing recognition that achievement of high predictive accuracy is likely to require prognostic models derived from patient characteristics that cover all influential domains of health [12]. For example, in musculoskeletal conditions, there is compelling evidence that psychological and social factors play important prognostic roles $[13,14]$. Increasingly therefore, prognostic research includes factors from such diverse domains as pain, activity limitation, physical impairment, work and social role participation, psychological factors, and biomedical testing (biomarkers and imaging).

However, such 'whole person' prognostic research may result in data-driven subgroups that are complex, difficult to interpret and challenging to recognise clinically. It would be useful if there were methodological approaches that harnessed the explanatory potential of that complexity while also facilitating clinical interpretability. An additional consideration is that modelling larger numbers of potentially predictive factors from multiple health domains requires larger patient sample sizes to avoid 'overfitting'. Overfitting is present when an analysis excessively fits the available data and therefore has limited generalisability outside the available sample [15]. So, it would also be useful if there were methodological approaches that harnessed the explanatory potential of many predictive variables while also minimising the need for very large patient samples.

The main aim of this paper was to describe a novel approach to using statistical clustering techniques to identify subgroups of patients, an approach that theoretically may improve clinical interpretability and reduce sample size requirements. We used Latent Class Analysis [16] 
as an exemplar statistical clustering technique and nonspecific low back pain as an exemplar health condition when explaining the conceptual approach. We chose low back pain because the potential usefulness of subgrouping in this condition is well recognised [17-19]. Subsequently, we used real chest pain data to illustrate a worked example, and chose these chest pain data because we had a suspicion that these data were likely to contain two latent subgroups. However, the approach may have potential application to other health conditions as well. The paper also suggests a framework for testing whether such subgroups are clinically important. The description of this novel approach is suggested as an option for researchers who use clustering techniques on complex clinical data and who have the intention of interpreting results in ways that are clinically meaningful.

\section{Methods}

Traditional approach to using statistical clustering techniques (low back pain example)

The traditional approach to using statistical clustering techniques to identify subgroups of patients is to enter selected clinical characteristics into a clustering model and then determine post-hoc in what ways the derived subgroups differ from each other. This general approach is illustrated in Fig. 1, which also shows how the same concept or attribute is named differently in clinical language and statistical language. To aid interpretability by clinically-orientated readers, this paper favours clinical language wherever possible.

For example, in a group of people with non-specific low back pain, we might select measures of various constructs such as pain (e.g. pain intensity, duration, number of previous episodes), activity limitation (difficulty in sit to stand, walking, lifting, rolling over in bed), physical impairments (lumbar spine movement, neurological signs), participation (sick leave, type of work), psychological factors (expectations of recovery, fear of movement, self efficacy, catastrophisation, depression), and social factors (income, partner support, social isolation). In this hypothetical example, the results might indicate that there are three subgroups that best explain how people in the sample scored on these characteristics (these clustering techniques aim to identify the subgroup structure that minimises the withinsubgroup variability and maximises the between-subgroup variability).

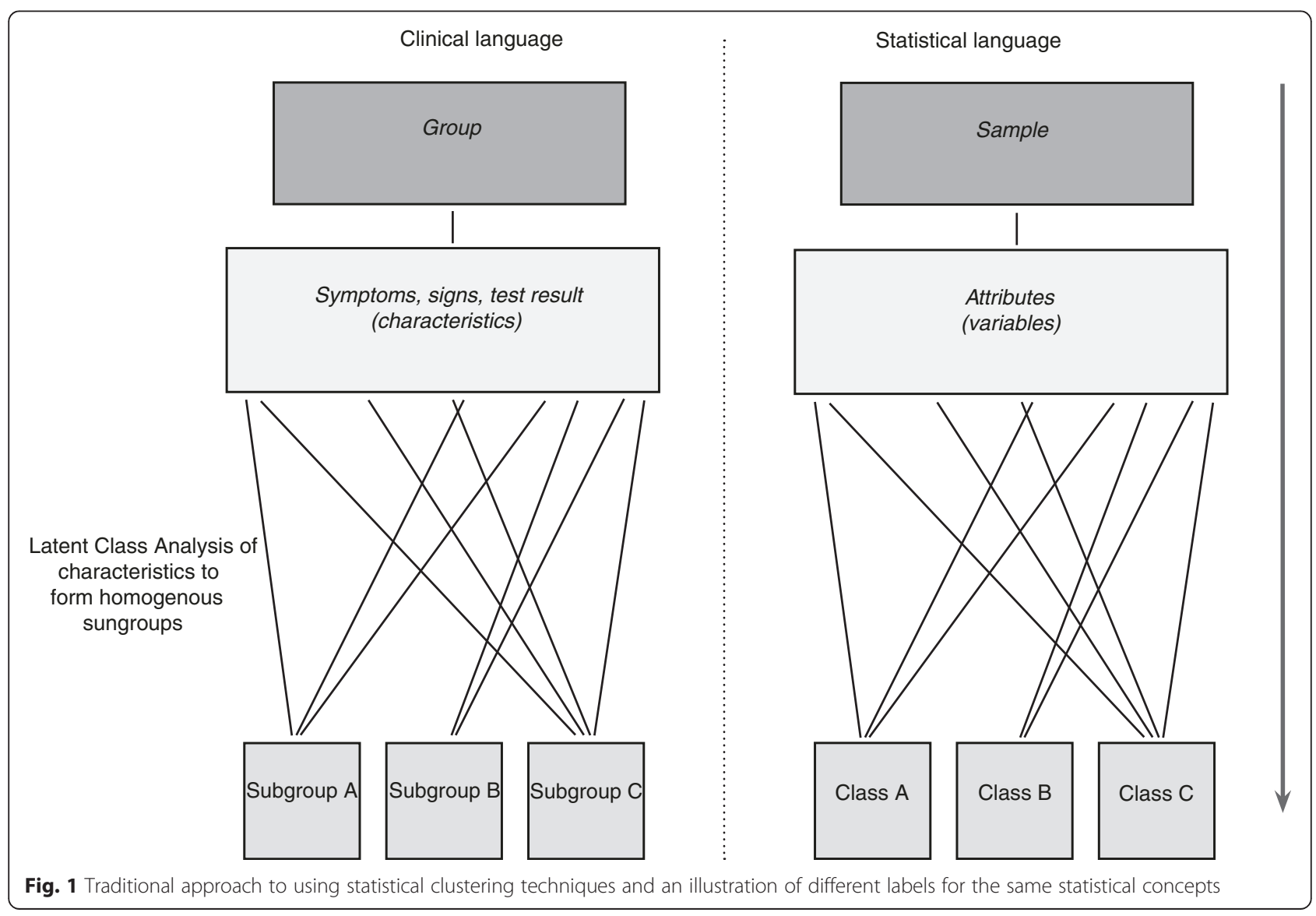


This approach is straightforward but as the number of clinical characteristics increases, interpretation and naming of the subgroup patterns of scoring can become more challenging. This is because with increasing complexity, the patterns may no longer be clinically recognisable. Moreover, the statistically optimal number of subgroups may become so high that it does not make practical sense.

\section{Novel approach of using statistical clustering techniques (low back pain example)}

The novel approach we suggest seeks to improve clinical interpretability and reduce sample size requirements by performing statistical clustering in two sequential stages. The first stage involves clustering using only clinical characteristics from within each health domain and therefore requires creating as many clustering models as there are health domains in the available data. This first stage produces scoring patterns within each domain. The second stage involves clustering using the scoring patterns from each health domain to identify subgroups across all the domains. Therefore, the scoring patterns identified in the first stage are treated as manifest categorical variables in the second stage. This principle is illustrated in Fig. 2.

\section{First stage clustering}

Initially, the researcher needs to classify each available clinical characteristic as belonging exclusively to one health domain. The International Classification of Functioning, Disability and Health from the World Health Organisation is an example of a classification tool that can guide this process [20]. Some clinical characteristics may be ambiguous to classify and in such cases, classification decisions will necessarily be arbitrary.

Next, clustering is performed using only clinical characteristics from within each health domain. So extending our example, Latent Class Analysis could be initially performed using only the pain characteristics (pain intensity, duration and number of previous episodes). The results of the Latent Class Analysis would identify how many discrete patterns of scoring on the pain characteristics best explained the variance in the whole sample. For example, one scoring pattern might be labelled 'high pain intensity/short duration' if it mainly varied from the other patterns on these two pain characteristics. This process of Latent Class Analysis, interpretation and labelling of results would then be repeated for each health domain.

Then, new categorical variables would be formed, one for each domain, with the categories (values) in each variable corresponding to the number of scoring patterns in that domain. For example, a new pain variable might be formed containing three categories that are labelled 'high pain intensity/short duration', 'high pain intensity/constant pain', and 'low pain intensity/long duration'.

One benefit of this first stage is potentially enhanced clinical interpretability of the results because the labelling only describes clinical characteristics from within one health domain and therefore each scoring pattern may be more recognisable. A second likely benefit is reduced sample size requirements because clustering within domains can be a powerful data-reduction technique. In our simple example, this first stage would have reduced the number of clinical characteristics (variables) being modelled from 16 (pain intensity, pain duration, pain behaviour, activity limitation, lumbar spine movement, neurological signs, sick leave, type of work, number of previous episodes, expectations of recovery, fear of movement, self efficacy, catastrophisation, depression, partner support and social isolation) to six (the domains of pain, activity limitation, participation, physical impairment, psychology, and social factors). That is because only the number of variables within each domain would be clustered during the first stage and only the number of variables that equals the number of domains would be clustered in the second stage. This data reduction would potentially be even greater in circumstances where some of the constructs are multi-dimensional and where greater numbers of clinical characteristics are being modelled.

\section{Second stage clustering}

The second stage involves clustering, using the categorical variables derived from the first stage, to identify subgroups across all the domains. So, in our example, we would perform a second Latent Class Analysis modelling the six variables that each represents the scoring patterns within one health domain. It is likely that, relative to the traditional method for performing statistical clustering, the clinical interpretability of the results would be enhanced because the subgroups would be formed from fewer variables that already contain values (scoring patterns) that have clinically interpretable labels.

An analogy for this novel approach to statistical clustering is the use of written language to explain a story (Fig. 2). We start with an idea (a latent story) that we wish to meaningfully explain. We require letters (variables) to form words (scoring patterns) that can be assembled into sentences (derived variables for each health domain) to explain the story (subgroups). The traditional method for performing statistical clustering attempts to bridge from letters (variables) through to the explained story (subgroups) and is, at least theoretically, subsequently more difficult to read. However, it may also result in a more interesting and unexpected story. 


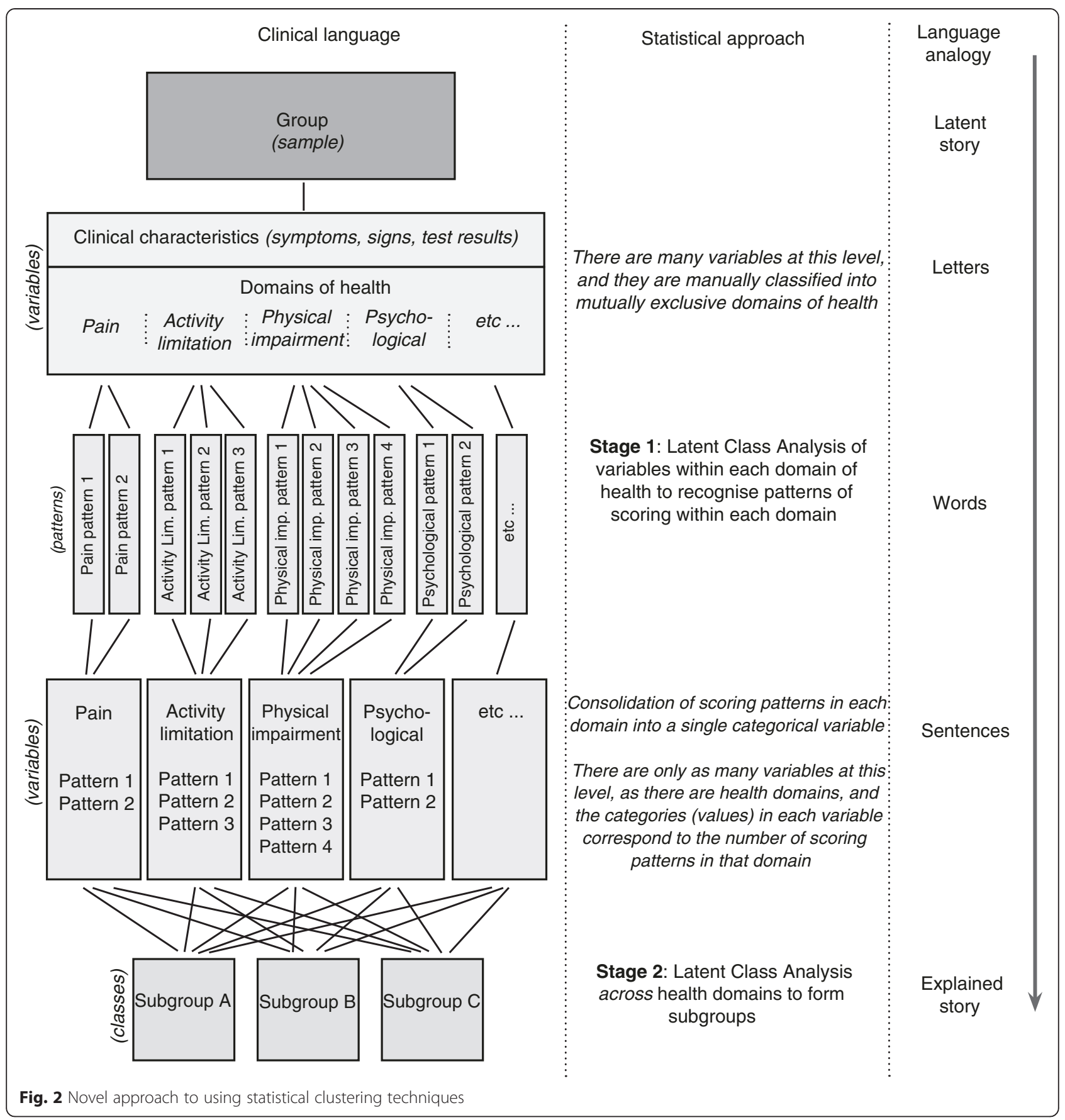

In summary, the potential advantages of this novel approach are enhanced clinical interpretability of dataderived subgroups and reduced sample size requirements. Potential disadvantages of this method are: (i) that it is more time-consuming to build separate clustering models for each included health domain and perform statistical clustering in two stages, (ii) the first stage clustering may hide potentially important interactions between factors from different domains.

\section{Assessing clinical importance}

To determine if these subgroups are clinically important due to their having prognostic or treatment effect implications, they would need to be tested for their association with clinical outcomes and some methods for doing so are shown in Fig. 3. Those methods are divided into three lines of inquiry: (i) did patients in each subgroup have different prognoses?, (ii) did patients in these subgroups respond differently to treatment?, and (iii) how 


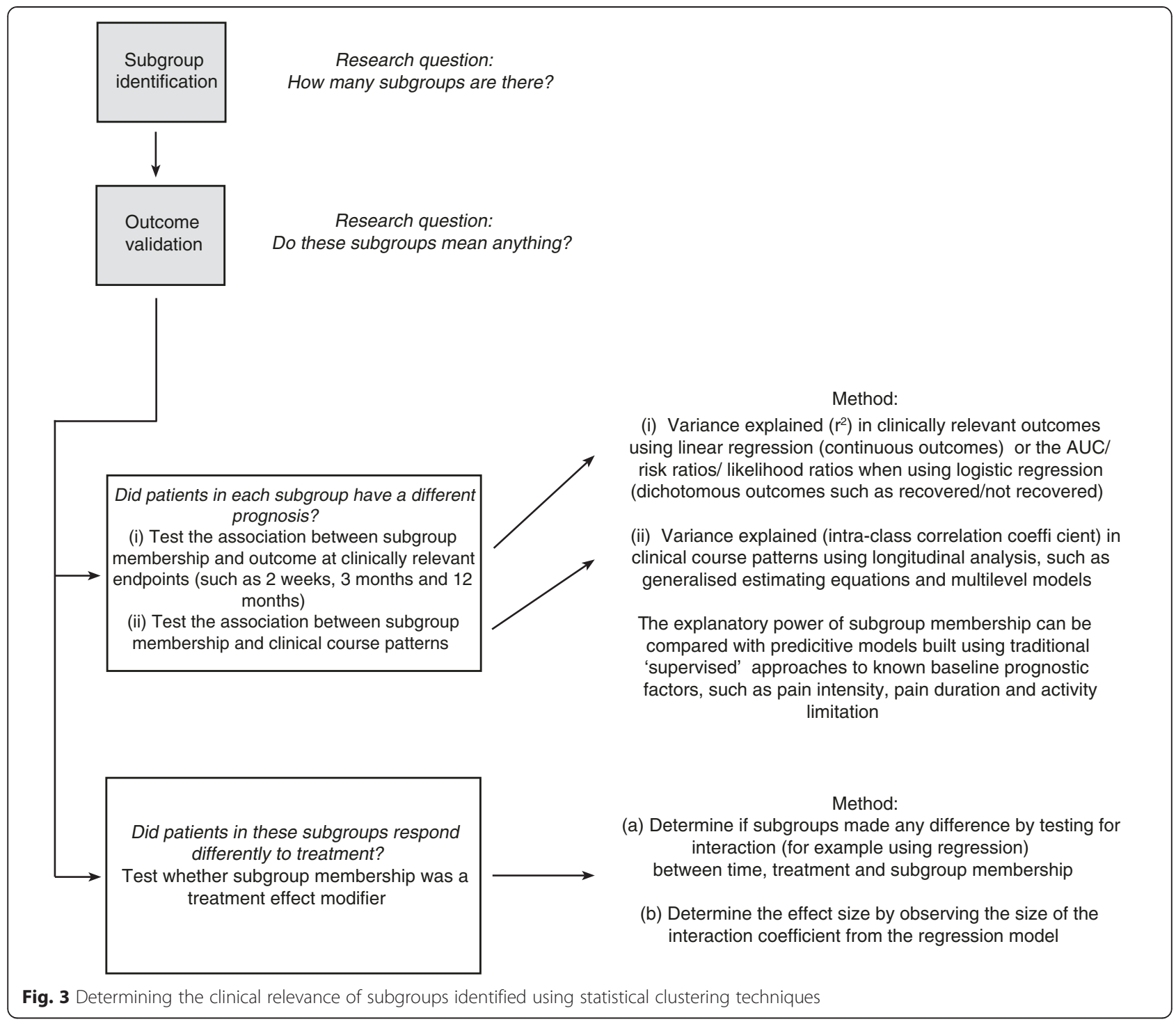

did the performance of this subgroup classification compare with other classification methods? This novel approach for applying statistical clustering techniques to identify clinically useful subgroups has potential benefits, but for any particular patient sample, the added value compared to the traditional approach needs to be evaluated. This can be tested by analysing the data using both approaches and comparing the interpretability of their results and the strength of their predictive capacity. For instance, either the traditional approach or the novel approach may explain more variance in clinically relevant outcomes and allow greater predictive accuracy when estimating the prognosis or treatment response of individual patients. So, all of the statistical approaches described in Fig. 3 can be used to also test whether one method for applying statistical clustering techniques is more useful in a given dataset. In the case of the chest pain data used as an example in the current study, we do not report whether the subgroups had prognostic or treatment effect implications, because the outcomes in the two data sources differed.

If statistically-derived subgroups show predictive validity at a clinically useful level, it can be helpful to then construct a clinical prediction rule that will allow clinicians to easily identify the subgroup membership of individual patients. This process requires identification of the most suitable set of clinical characteristics and creation of a scoring algorithm capable of allocating patients to the correct subgroup, while balancing operational simplicity with classification accuracy. There are a number of methods for doing this. Three examples of these methods are (i) the use of receiver operating characteristic (ROC) 
curve analysis and contingency tables [9], (ii) the use of logistic regression and scoring tables [8], and (iii) the use of more automated techniques such as Classification and Regression Tree Analysis [21].

Lastly, the type of clustering approaches described in this paper are hypothesis-setting, even when predictive accuracy has been demonstrated in an initial patient sample. There are subsequent stages of research needed to externally validate new subgrouping tools and adequately test their capacity to improve clinical effects and/or increase health system efficiency $[9,22]$.

\section{Results}

\section{Example of this method applied (non-specific chest pain example)}

Identical baseline variables from two clinical trials of non-specific chest pain in Denmark were combined to create test data on which to apply this method of analysis. The researchers working with these data had a suspicion that there may be two latent subgroups, one subgroup representing predominantly cardiogenic chest pain, and the other representing predominantly musculoskeletal chest pain.

One clinical trial was of two treatments for acute musculoskeletal chest pain undertaken in an emergency cardiology department and four chiropractic clinics, and the data from evaluating 305 prospective participants was included in the current study. Full details of the study design and recruitment procedures have been published previously $[23,24]$. The other clinical trial was of manual therapy for chest pain in people diagnosed with or without cervico-thoracic angina, and the data from evaluating 275 prospective participants was included in the current study. Full details of that study's design and recruitment procedures have also been published previously $[25,26]$. Permission was obtained from the custodians (MJS and HWC) of each of these datasets for secondary use of the data within this project. Under Danish law, the secondary analysis of such de-identified data does not require separate ethics approval (The Act on Processing of Personal Data, December 2012, Section 5.2; Act on Research Ethics Review of Health Research Projects, October 2013, Section 14.2).

For both the single-stage and two-stage clustering, 69 variables were selected that traversed the health domains of demographics, previous history, psychological perception and coping, pain, activity limitation, diagnostic classification and musculoskeletal palpation findings, so as to illustrate data-derived subgroups that were identified using information across health domains. The number of variables differed across domains and also contained a variety of metrics. A detailed description of all variables is shown in Table 1 . All variables contained $1 \%$ or less missing data, except for the CCS angina classification variable (1.6\%), SF36 physical function sum score $(3.5 \%)$ and average chest pain episode duration (21.9\%). However, no data were imputed, as Latent Class Analysis copes with missing data.

Latent Class Analysis was performed using Latent Gold (version 4.5, Statistical Innovations, Belmont MA, $U S A$ ), and as use of a random seed start-point in the data can produce some slight variability in results, all analyses were performed five times. The appropriate number of clusters (subgroups) was chosen from the model with the lowest and most consistent Bayesian Information Criterion (BIC) across the five repetitions.

All subgroups and scoring patterns were independently named by two researchers (MJS and HWC) with content expertise and then a consensus was reached by discussion. The two experts had conducted the clinical trials and were very familiar with both the variables and the clinical context in which the trials occurred.

The results from the single-stage clustering are shown in Table 2. Five subgroups were identified which were subsequently named: (i) Low activity limitation, high fear and few palpation findings (32\% of sample), (ii) Typical and atypical angina, short episode, with pectoral tenderness (26\%), (iii) no palpation findings (24\%), (iv) $\mathrm{Fe}$ male, high fear, diffuse anterior tenderness (10\%), and (v) Typical angina, short episode, high activity limitation, with pectoral tenderness (7\%).

The results from the first stage of the two-stage clustering are shown in Table 3, including the names of the two to six scoring patterns that were identified within each health domain. The results from the second stage are shown in Tables 4 and 5, which identified two subgroups which were subsequently named: (i) Uncertain of cause and fearful, but not heart-related - with local thoracic 5/6 palpation findings (51\% of sample), and (ii) Believes pain to be heart- or musculoskeletal-related with crushing pain, local thoracic $2 / 3$ signs, and pectoral tenderness (49\%). In Table 4, these two subgroups are described using the proportions of individuals in each of the first stage subgroups. In Table 5, these two subgroups are described in the measurement units of the original variables.

A cross-tabulation of the cluster membership between the five subgroups from single-stage clustering and the two subgroups from two-stage clustering is shown in Table 6. One of the subgroups from the single-stage clustering was split across the two subgroups from two-stage clustering, but the rest of the single-stage subgroups were largely represented in only one of the two-stage subgroups.

\section{Discussion}

We have introduced a novel approach to using statistical clustering techniques to identify clinically useful 
Table 1 Description of variables

Demographics domain

Age

Gender

Previous history domain

Risk factors for cardiovascular disease sum score of hypercholesterolemia, hypertension, 0 to 5 diabetes, family history of cardiac disease, previous or current smoking (all scored yes/no)

Psychological domain (patient perception and coping)

Does the pain originate from the heart?

Yes/possibly/no $\quad 0.4 \%$

Does the pain originate from the muscles and joints?

Yes/possibly/no

Are you afraid?

Yes/no

Pain domain

Average chest pain episode duration

$<10 \mathrm{~min} / 10 \mathrm{~min}-1$ hour/> 1 hour/continuous

$21.9 \%$

Pain description 'crushing pain'

Yes/no

Pain description 'tenderness'

Yes/no

$0 \%$

Pain description 'sharp pains'

Yes/no

$0 \%$

Pain description 'well defined'

Yes/no

$0 \%$

Pain description 'diffuse'

Yes/no

$0 \%$

Pain description 'burning'

Yes/no

$0 \%$

Pain description 'tingling'

Yes/no

$0 \%$

Activity limitation domain

SF36 physical function sum score

0 to 100 scale (high scores better)

Diagnostic classification domain

Danish Cardiologist's Society classification 'angina'

No angina/typical/atypical/

Canadian Cardiovascular Society classification uncharacteristic/ unstable

Palpation domain

Grades 0 to 3

Anterior

Springing - tenderness sterno/xiphoid junction

Springing - tenderness costosternal 2-3 right

Springing - tenderness costosternal 2-3 left

Springing - tenderness costosternal 3-4 right

Springing - tenderness costosternal 3-4 left

Springing - tenderness costosternal 4-5 right

Springing - tenderness costosternal 4-5 left

Yes/no

$<1 \%$

Yes/no

$<1 \%$

Yes/no

$<1 \%$

Yes/no

$<1 \%$

Springing - tenderness costosternal 5-6 right

Yes/no

$<1 \%$

Yes/no

$<1 \%$

Yes/no

$<1 \%$

Springing - tenderness costosternal 5-6 left

Yes/no

$<1 \%$

Springing - provoked pain sterno/xiphoid junction

Yes/no

$<1 \%$

Springing - provoked pain costosternal 2-3 right

Yes/no

$<1 \%$

Springing - provoked pain costosternal 2-3 left

Yes/no

$<1 \%$

Springing - provoked pain costosternal 3-4 right

Yes/no

$<1 \%$

Springing - provoked pain costosternal 3-4 left

Yes/no

$<1 \%$

Springing - provoked pain costosternal 4-5 right

Yes/no

$<1 \%$

Springing - provoked pain costosternal 4-5 left

Yes/no

$<1 \%$

Yes/no 
Table 1 Description of variables (Continued)

Springing - provoked pain costosternal 5-6 right

Springing - provoked pain costosternal 5-6 left

Muscle tenderness/pain pectoralis maj. left

Muscle tenderness/pain pectoralis min. left

Muscle tenderness/pain intercostalis 2-3 left

Muscle tenderness/pain intercostalis 3-4 left

Muscle tenderness/pain intercostalis 4-5 left

Muscle tenderness/pain intercostalis 5-6 left

Muscle tenderness/pain intercostalis 6-7 left

Muscle tenderness/pain pectoralis maj. right

Muscle tenderness/pain pectoralis min. right

Muscle tenderness/pain intercostalis 2-3 right

Muscle tenderness/pain intercostalis 3-4 right

Muscle tenderness/pain intercostalis 4-5 right

Muscle tenderness/pain intercostalis 5-6 right

Muscle tenderness/pain intercostalis 6-7 right

Posterior

Tender c4-5 paraspinalpost either left or right

Tender c5-6 paraspinalpost either left or right

Tender c6-7 paraspinalpost either left or right

Tender c7-th1 paraspinalpost left or right

Tender th1-2 paraspinalpost left or right

Tender th2-3 paraspinalpost left or right

Tender th3-4 paraspinalpost left or right

Tender th4-5 paraspinalpost left or right

Tender th5-6 paraspinalpost left or right

Tender th6-7 paraspinalpost left or right

Tender th7-8 paraspinalpost left or right

Tender th8-9 paraspinalpost left or right

Vertebral springing th1-2

Vertebral springing th2-3

Vertebral springing th3-4

Vertebral springing th4-5

Vertebral springing th5-6

Vertebral springing th6-7

Vertebral springing th7-8

Vertebral springing th8-9

\begin{tabular}{|c|c|}
\hline Yes/no & $<1 \%$ \\
\hline Yes/no & $<1 \%$ \\
\hline No tenderness/tenderness/pain & $<1 \%$ \\
\hline No tenderness/tenderness/pain & $<1 \%$ \\
\hline No tenderness/tenderness/pain & $<1 \%$ \\
\hline No tenderness/tenderness/pain & $<1 \%$ \\
\hline No tenderness/tenderness/pain & $<1 \%$ \\
\hline No tenderness/tenderness/pain & $<1 \%$ \\
\hline No tenderness/tenderness/pain & $<1 \%$ \\
\hline No tenderness/tenderness/pain & $<1 \%$ \\
\hline No tenderness/tenderness/pain & $<1 \%$ \\
\hline No tenderness/tenderness/pain & $<1 \%$ \\
\hline No tenderness/tenderness/pain & $<1 \%$ \\
\hline No tenderness/tenderness/pain & $<1 \%$ \\
\hline No tenderness/tenderness/pain & $<1 \%$ \\
\hline No tenderness/tenderness/pain & $<1 \%$ \\
\hline Yes/no & $1 \%$ \\
\hline Yes/no & $1 \%$ \\
\hline Yes/no & $1 \%$ \\
\hline Yes/no & $1 \%$ \\
\hline Yes/no & $1 \%$ \\
\hline Yes/no & $1 \%$ \\
\hline Yes/no & $1 \%$ \\
\hline Yes/no & $1 \%$ \\
\hline Yes/no & $1 \%$ \\
\hline Yes/no & $1 \%$ \\
\hline Yes/no & $1 \%$ \\
\hline Yes/no & $1 \%$ \\
\hline Yes/no & $1 \%$ \\
\hline Yes/no & $1 \%$ \\
\hline Yes/no & $1 \%$ \\
\hline Yes/no & $1 \%$ \\
\hline Yes/no & $1 \%$ \\
\hline Yes/no & $1 \%$ \\
\hline Yes/no & $1 \%$ \\
\hline Yes/no & $1 \%$ \\
\hline
\end{tabular}

subgroups of patients. It has been described using the hypothetical example of low back pain and then illustrated using an applied example of chest pain data. From those cross-sectional chest pain data, we reported the baseline subgroups detected using the traditional approach and the baseline subgroups detected using the novel approach.
We anticipated that two-stage clustering might result in more clinically interpretable subgrouping than traditional one-stage clustering, due to its giving equal potential weight to variables from each domain. In this chest pain sample, the two approaches resulted in two and five subgroups respectively, both of which appeared clinically interpretable and reasonably recognisable. The 
Table 2 Results of the single-stage clustering of chest pain data

\begin{tabular}{|c|c|c|c|c|c|c|}
\hline & $\begin{array}{l}\text { Response } \\
\text { option }\end{array}$ & Cluster1 & Cluster2 & Cluster3 & Cluster4 & Cluster5 \\
\hline Subgroup label & & $\begin{array}{l}\text { Low activity limitation, } \\
\text { high fear, few } \\
\text { palpation findings }\end{array}$ & $\begin{array}{l}\text { Typical and atypical } \\
\text { angina, short episode, } \\
\text { pectoral tenderness }\end{array}$ & $\begin{array}{l}\text { No } \\
\text { palpation } \\
\text { findings }\end{array}$ & $\begin{array}{l}\text { Female, high fear, } \\
\text { diffuse anterior } \\
\text { tenderness }\end{array}$ & $\begin{array}{l}\text { Typical angina, short episode, } \\
\text { high activity limitation, } \\
\text { pectoral tenderness }\end{array}$ \\
\hline Cluster Size & & $32 \%$ & $26 \%$ & $24 \%$ & $10 \%$ & $7 \%$ \\
\hline $\begin{array}{l}\text { Cluster } \\
\text { membership } \\
\text { probability, }\end{array}$ & $\begin{array}{l}\text { Median } \\
\text { (interquartile } \\
\text { range) }\end{array}$ & $\begin{array}{l}100 \%(100 \% \text { to } \\
100 \%)\end{array}$ & $100 \%(100 \%$ to $100 \%)$ & $\begin{array}{l}100 \% \\
(100 \% \text { to } \\
100 \%)\end{array}$ & $\begin{array}{l}100 \%(100 \% \text { to } \\
100 \%)\end{array}$ & $100 \%(100 \%$ to $100 \%)$ \\
\hline \multicolumn{7}{|l|}{$\begin{array}{l}\text { Demographic } \\
\text { domain }\end{array}$} \\
\hline Age & Mean (SD) & $51.5(11.0)$ & $55.4(9.4)$ & $55.3(10.3)$ & $54.5(10.2)$ & $59.0(9.0)$ \\
\hline Gender & Female & $42 \%$ & $33 \%$ & $27 \%$ & $93 \%$ & $36 \%$ \\
\hline \multicolumn{7}{|l|}{$\begin{array}{l}\text { Previous history } \\
\text { domain }\end{array}$} \\
\hline $\begin{array}{l}\text { Cardiovascular } \\
\text { risk factor Index }\end{array}$ & Mean (SD) & $1.9(1.1)$ & $2.1(1.1)$ & $2.0(1.2)$ & $2.1(1.1)$ & $2.4(0.9)$ \\
\hline \multicolumn{7}{|l|}{$\begin{array}{l}\text { Psychological } \\
\text { domain }\end{array}$} \\
\hline \multirow{2}{*}{$\begin{array}{l}\text { Self-perceived } \\
\text { 'Pain from } \\
\text { muscle or joints' }\end{array}$} & Possibly & $43 \%$ & $42 \%$ & $36 \%$ & $41 \%$ & $43 \%$ \\
\hline & Yes & $31 \%$ & $32 \%$ & $12 \%$ & $38 \%$ & $30 \%$ \\
\hline \multirow{2}{*}{$\begin{array}{l}\text { Self-perceived } \\
\text { 'Pain from heart' }\end{array}$} & Possibly & $39 \%$ & $35 \%$ & $37 \%$ & $40 \%$ & $32 \%$ \\
\hline & Yes & $22 \%$ & $52 \%$ & $47 \%$ & $29 \%$ & $59 \%$ \\
\hline $\begin{array}{l}\text { Self-perceived } \\
\text { 'Afraid' }\end{array}$ & Yes & $94 \%$ & $69 \%$ & $77 \%$ & $92 \%$ & $82 \%$ \\
\hline \multicolumn{7}{|l|}{ Pain domain } \\
\hline \multirow[t]{4}{*}{ Episode duration } & $<10 \min$ & $38 \%$ & $72 \%$ & $59 \%$ & $50 \%$ & $68 \%$ \\
\hline & 10 min-1 hour & $20 \%$ & $18 \%$ & $21 \%$ & $21 \%$ & $19 \%$ \\
\hline & $>1$ hour & $9 \%$ & $4 \%$ & $6 \%$ & $7 \%$ & $4 \%$ \\
\hline & Continuous & $34 \%$ & $7 \%$ & $14 \%$ & $22 \%$ & $9 \%$ \\
\hline $\begin{array}{l}\text { Pain description } \\
\text { 'crushing pain' }\end{array}$ & Yes & $73 \%$ & $92 \%$ & $80 \%$ & $82 \%$ & $92 \%$ \\
\hline $\begin{array}{l}\text { Pain description } \\
\text { 'tenderness' }\end{array}$ & Yes & $73 \%$ & $73 \%$ & $73 \%$ & $73 \%$ & $73 \%$ \\
\hline $\begin{array}{l}\text { Pain description } \\
\text { 'sharp pains' }\end{array}$ & Yes & $29 \%$ & $18 \%$ & $16 \%$ & $28 \%$ & $26 \%$ \\
\hline $\begin{array}{l}\text { Pain description } \\
\text { 'well defined' }\end{array}$ & Yes & $4 \%$ & $4 \%$ & $3 \%$ & $3 \%$ & $5 \%$ \\
\hline $\begin{array}{l}\text { Pain description } \\
\text { 'diffuse' }\end{array}$ & Yes & $4 \%$ & $10 \%$ & $3 \%$ & $2 \%$ & $8 \%$ \\
\hline $\begin{array}{l}\text { Pain description } \\
\text { 'burning' }\end{array}$ & Yes & $7 \%$ & $4 \%$ & $4 \%$ & $8 \%$ & $5 \%$ \\
\hline $\begin{array}{l}\text { Pain description } \\
\text { 'tingling' }\end{array}$ & Yes & $7 \%$ & $17 \%$ & $9 \%$ & $7 \%$ & $10 \%$ \\
\hline \multicolumn{7}{|l|}{$\begin{array}{l}\text { Activity } \\
\text { limitation } \\
\text { domain }\end{array}$} \\
\hline $\begin{array}{l}\text { SF36 physical } \\
\text { function } \\
\text { sum-score }\end{array}$ & Mean (SD) & $70.8(9.9)$ & $67.0(9.4)$ & 66.8 (11.9) & $58.9(16.2)$ & $28.6(8.9)$ \\
\hline
\end{tabular}


Table 2 Results of the single-stage clustering of chest pain data (Continued)

\begin{tabular}{|c|c|c|c|c|c|c|}
\hline \multicolumn{7}{|l|}{$\begin{array}{l}\text { Diagnostic } \\
\text { classification } \\
\text { domain }\end{array}$} \\
\hline \multirow{5}{*}{$\begin{array}{l}\text { Danish } \\
\text { Cardiologist's } \\
\text { Society } \\
\text { classification } \\
\text { 'angina' }\end{array}$} & No angina & $45 \%$ & $3 \%$ & $23 \%$ & $38 \%$ & $8 \%$ \\
\hline & Typical & $9 \%$ & $52 \%$ & $31 \%$ & $27 \%$ & $77 \%$ \\
\hline & Atypical & $17 \%$ & $32 \%$ & $24 \%$ & $15 \%$ & $6 \%$ \\
\hline & Uncharacteristic & $30 \%$ & $14 \%$ & $21 \%$ & $20 \%$ & $10 \%$ \\
\hline & Unstable & $0 \%$ & $0 \%$ & $1 \%$ & $0 \%$ & $0 \%$ \\
\hline \multirow{4}{*}{$\begin{array}{l}\text { Canadian } \\
\text { Cardiovascular } \\
\text { Society } \\
\text { classification }\end{array}$} & Grade 0 & $65 \%$ & $12 \%$ & $27 \%$ & $35 \%$ & $3 \%$ \\
\hline & Grade 1 & $29 \%$ & $35 \%$ & $42 \%$ & $41 \%$ & $21 \%$ \\
\hline & Grade 2 & $6 \%$ & $48 \%$ & $30 \%$ & $23 \%$ & $63 \%$ \\
\hline & Grade 3 & $0 \%$ & $4 \%$ & $1 \%$ & $1 \%$ & $12 \%$ \\
\hline \multicolumn{7}{|l|}{ Palpation domain } \\
\hline $\begin{array}{l}\text { Sternoxiphoid } \\
\text { springing } \\
\text { tenderness }\end{array}$ & Yes & $16 \%$ & $1 \%$ & $9 \%$ & $38 \%$ & $0 \%$ \\
\hline $\begin{array}{l}\text { Costosternal } \\
\text { springing } \\
\text { tenderness } \\
2 / 3 \text { right }\end{array}$ & Yes & $11 \%$ & $2 \%$ & $0 \%$ & $42 \%$ & $5 \%$ \\
\hline $\begin{array}{l}\text { Costosternal } \\
\text { springing } \\
\text { tenderness } \\
2 / 3 \text { left }\end{array}$ & Yes & $15 \%$ & $1 \%$ & $0 \%$ & $45 \%$ & $3 \%$ \\
\hline $\begin{array}{l}\text { Costosternal } \\
\text { springing } \\
\text { tenderness } \\
3 / 4 \text { right }\end{array}$ & Yes & $14 \%$ & $0 \%$ & $1 \%$ & $45 \%$ & $5 \%$ \\
\hline $\begin{array}{l}\text { Costosternal } \\
\text { springing } \\
\text { tenderness } \\
3 / 4 \text { left }\end{array}$ & Yes & $22 \%$ & $0 \%$ & $0 \%$ & $52 \%$ & $3 \%$ \\
\hline $\begin{array}{l}\text { Costosternal } \\
\text { springing } \\
\text { tenderness } \\
4 / 5 \text { right }\end{array}$ & Yes & $15 \%$ & $0 \%$ & $1 \%$ & $57 \%$ & $3 \%$ \\
\hline $\begin{array}{l}\text { Costosternal } \\
\text { springing } \\
\text { tenderness } \\
4 / 5 \text { left }\end{array}$ & Yes & $45 \%$ & $0 \%$ & $1 \%$ & $62 \%$ & $5 \%$ \\
\hline $\begin{array}{l}\text { Costosternal } \\
\text { springing } \\
\text { tenderness } \\
5 / 6 \text { right }\end{array}$ & Yes & $11 \%$ & $1 \%$ & $1 \%$ & $57 \%$ & $3 \%$ \\
\hline $\begin{array}{l}\text { Costosternal } \\
\text { springing } \\
\text { tenderness } \\
5 / 6 \text { left }\end{array}$ & Yes & $30 \%$ & $1 \%$ & $3 \%$ & $59 \%$ & $0 \%$ \\
\hline $\begin{array}{l}\text { sternoxiphoid } \\
\text { springing pain }\end{array}$ & Yes & $3 \%$ & $0 \%$ & $3 \%$ & $7 \%$ & $0 \%$ \\
\hline $\begin{array}{l}\text { Costosternal } \\
\text { springing pain } \\
2 / 3 \text { right }\end{array}$ & Yes & $2 \%$ & $1 \%$ & $0 \%$ & $7 \%$ & $2 \%$ \\
\hline
\end{tabular}


Table 2 Results of the single-stage clustering of chest pain data (Continued)

\begin{tabular}{|c|c|c|c|c|c|c|}
\hline $\begin{array}{l}\text { Costosternal } \\
\text { springing pain } \\
2 / 3 \text { left }\end{array}$ & Yes & $3 \%$ & $1 \%$ & $0 \%$ & $8 \%$ & $0 \%$ \\
\hline $\begin{array}{l}\text { Costosternal } \\
\text { springing pain } \\
3 / 4 \text { right }\end{array}$ & Yes & $3 \%$ & $0 \%$ & $1 \%$ & $9 \%$ & $2 \%$ \\
\hline $\begin{array}{l}\text { Costosternal } \\
\text { springing pain } \\
3 / 4 \text { left }\end{array}$ & Yes & $8 \%$ & $0 \%$ & $0 \%$ & $8 \%$ & $0 \%$ \\
\hline $\begin{array}{l}\text { Costosternal } \\
\text { springing pain } \\
4 / 5 \text { right }\end{array}$ & Yes & $3 \%$ & $0 \%$ & $0 \%$ & $14 \%$ & $2 \%$ \\
\hline $\begin{array}{l}\text { Costosternal } \\
\text { springing pain } \\
4 / 5 \text { left }\end{array}$ & Yes & $19 \%$ & $0 \%$ & $0 \%$ & $12 \%$ & $0 \%$ \\
\hline $\begin{array}{l}\text { Costosternal } \\
\text { springing pain } \\
5 / 6 \text { right }\end{array}$ & Yes & $3 \%$ & $0 \%$ & $0 \%$ & $10 \%$ & $0 \%$ \\
\hline $\begin{array}{l}\text { Costosternal } \\
\text { springing pain } \\
5 / 6 \text { left }\end{array}$ & Yes & $16 \%$ & $0 \%$ & $0 \%$ & $12 \%$ & $0 \%$ \\
\hline \multirow{2}{*}{$\begin{array}{l}\text { Muscle } \\
\text { tenderness } \\
\text { pectoralis major } \\
\text { left }\end{array}$} & Tenderness & $26 \%$ & $72 \%$ & $8 \%$ & $47 \%$ & $61 \%$ \\
\hline & Pain & $4 \%$ & $23 \%$ & $2 \%$ & $18 \%$ & $22 \%$ \\
\hline \multirow{2}{*}{$\begin{array}{l}\text { Muscle } \\
\text { tenderness } \\
\text { pectoralis minor } \\
\text { left }\end{array}$} & Tenderness & $34 \%$ & $82 \%$ & $4 \%$ & $61 \%$ & $77 \%$ \\
\hline & Pain & $6 \%$ & $11 \%$ & $1 \%$ & $15 \%$ & $8 \%$ \\
\hline \multirow{2}{*}{$\begin{array}{l}\text { Muscle } \\
\text { tenderness } \\
\text { intercostal 2/3 } \\
\text { left }\end{array}$} & Tenderness & $15 \%$ & $50 \%$ & $1 \%$ & $69 \%$ & $55 \%$ \\
\hline & Pain & $0 \%$ & $3 \%$ & $0 \%$ & $8 \%$ & $5 \%$ \\
\hline \multirow{2}{*}{$\begin{array}{l}\text { Muscle } \\
\text { tenderness } \\
\text { intercostal 3/4 } \\
\text { left }\end{array}$} & Tenderness & $20 \%$ & $30 \%$ & $0 \%$ & $80 \%$ & $25 \%$ \\
\hline & Pain & $2 \%$ & $0 \%$ & $0 \%$ & $7 \%$ & $2 \%$ \\
\hline \multirow{2}{*}{$\begin{array}{l}\text { Muscle } \\
\text { tenderness } \\
\text { intercostal 4/5 } \\
\text { left }\end{array}$} & Tenderness & $26 \%$ & $13 \%$ & $1 \%$ & $86 \%$ & $13 \%$ \\
\hline & Pain & $8 \%$ & $1 \%$ & $0 \%$ & $10 \%$ & $2 \%$ \\
\hline \multirow{2}{*}{$\begin{array}{l}\text { Muscle } \\
\text { tenderness } \\
\text { intercostal 5/6 } \\
\text { left }\end{array}$} & Tenderness & $33 \%$ & $5 \%$ & $2 \%$ & $78 \%$ & $5 \%$ \\
\hline & Pain & $9 \%$ & $0 \%$ & $0 \%$ & $15 \%$ & $0 \%$ \\
\hline \multirow{2}{*}{$\begin{array}{l}\text { Muscle } \\
\text { tenderness } \\
\text { intercostal 6/7 } \\
\text { left }\end{array}$} & Tenderness & $22 \%$ & $1 \%$ & $1 \%$ & $75 \%$ & $3 \%$ \\
\hline & Pain & $7 \%$ & $0 \%$ & $0 \%$ & $13 \%$ & $0 \%$ \\
\hline \multirow{2}{*}{$\begin{array}{l}\text { Muscle } \\
\text { tenderness } \\
\text { pectoralis major } \\
\text { right }\end{array}$} & Tenderness & $20 \%$ & $71 \%$ & $9 \%$ & $49 \%$ & $73 \%$ \\
\hline & Pain & $2 \%$ & $22 \%$ & $0 \%$ & $18 \%$ & $13 \%$ \\
\hline \multirow{2}{*}{$\begin{array}{l}\text { Muscle } \\
\text { tenderness } \\
\text { pectoralis minor } \\
\text { right }\end{array}$} & Tenderness & $23 \%$ & $76 \%$ & $3 \%$ & $60 \%$ & $73 \%$ \\
\hline & Pain & $1 \%$ & $9 \%$ & $0 \%$ & $15 \%$ & $12 \%$ \\
\hline \multirow{2}{*}{$\begin{array}{l}\text { Muscle } \\
\text { tenderness } \\
\text { intercostal 2/3 } \\
\text { right }\end{array}$} & Tenderness & $13 \%$ & $48 \%$ & $0 \%$ & $76 \%$ & $54 \%$ \\
\hline & Pain & $1 \%$ & $0 \%$ & $0 \%$ & $7 \%$ & $2 \%$ \\
\hline
\end{tabular}


Table 2 Results of the single-stage clustering of chest pain data (Continued)

\begin{tabular}{|c|c|c|c|c|c|c|}
\hline \multirow{2}{*}{$\begin{array}{l}\text { Muscle } \\
\text { tenderness } \\
\text { intercostal 3/4 } \\
\text { right }\end{array}$} & Tenderness & $10 \%$ & $25 \%$ & $1 \%$ & $81 \%$ & $25 \%$ \\
\hline & Pain & $1 \%$ & $0 \%$ & $0 \%$ & $5 \%$ & $2 \%$ \\
\hline \multirow{2}{*}{$\begin{array}{l}\text { Muscle } \\
\text { tenderness } \\
\text { intercostal 4/5 } \\
\text { right }\end{array}$} & Tenderness & $12 \%$ & $9 \%$ & $0 \%$ & $88 \%$ & $5 \%$ \\
\hline & Pain & $0 \%$ & $0 \%$ & $0 \%$ & $7 \%$ & $0 \%$ \\
\hline \multirow{2}{*}{$\begin{array}{l}\text { Muscle } \\
\text { tenderness } \\
\text { intercostal 5/6 } \\
\text { right }\end{array}$} & Tenderness & $14 \%$ & $4 \%$ & $0 \%$ & $82 \%$ & $0 \%$ \\
\hline & Pain & $0 \%$ & $0 \%$ & $0 \%$ & $10 \%$ & $0 \%$ \\
\hline \multirow{2}{*}{$\begin{array}{l}\text { Muscle } \\
\text { tenderness } \\
\text { intercostal 6/7 } \\
\text { right }\end{array}$} & Tenderness & $8 \%$ & $2 \%$ & $0 \%$ & $79 \%$ & $0 \%$ \\
\hline & Pain & $1 \%$ & $0 \%$ & $0 \%$ & $10 \%$ & $0 \%$ \\
\hline $\begin{array}{l}\text { Tenderness c4/5 } \\
\text { paraspinal } \\
\text { posterior }\end{array}$ & Yes & $10 \%$ & $5 \%$ & $2 \%$ & $27 \%$ & $3 \%$ \\
\hline $\begin{array}{l}\text { Tenderness c5/6 } \\
\text { paraspinal } \\
\text { posterior }\end{array}$ & Yes & $16 \%$ & $8 \%$ & $4 \%$ & $26 \%$ & $3 \%$ \\
\hline $\begin{array}{l}\text { Tenderness c6/7 } \\
\text { paraspinal } \\
\text { posterior }\end{array}$ & Yes & $14 \%$ & $5 \%$ & $3 \%$ & $17 \%$ & $3 \%$ \\
\hline $\begin{array}{l}\text { Tenderness C7/ } \\
\text { th1 paraspinal } \\
\text { posterior }\end{array}$ & Yes & $7 \%$ & $11 \%$ & $1 \%$ & $18 \%$ & $10 \%$ \\
\hline $\begin{array}{l}\text { Tenderness th1/ } \\
2 \text { paraspinal } \\
\text { posterior }\end{array}$ & Yes & $14 \%$ & $27 \%$ & $3 \%$ & $29 \%$ & $38 \%$ \\
\hline $\begin{array}{l}\text { Tenderness th2/ } \\
3 \text { paraspinal } \\
\text { posterior }\end{array}$ & Yes & $29 \%$ & $50 \%$ & $5 \%$ & $50 \%$ & $64 \%$ \\
\hline $\begin{array}{l}\text { Tenderness th3/ } \\
4 \text { paraspinal } \\
\text { posterior }\end{array}$ & Yes & $37 \%$ & $46 \%$ & $3 \%$ & $58 \%$ & $47 \%$ \\
\hline $\begin{array}{l}\text { Tenderness th4/ } \\
5 \text { paraspinal } \\
\text { posterior }\end{array}$ & Yes & $45 \%$ & $24 \%$ & $3 \%$ & $56 \%$ & $29 \%$ \\
\hline $\begin{array}{l}\text { Tenderness th5/ } \\
6 \text { paraspinal } \\
\text { posterior }\end{array}$ & Yes & $46 \%$ & $9 \%$ & $2 \%$ & $45 \%$ & $13 \%$ \\
\hline $\begin{array}{l}\text { Tenderness th6/ } \\
7 \text { paraspinal } \\
\text { posterior }\end{array}$ & Yes & $42 \%$ & $2 \%$ & $1 \%$ & $40 \%$ & $5 \%$ \\
\hline $\begin{array}{l}\text { Tenderness th7/ } \\
8 \text { paraspinal } \\
\text { posterior }\end{array}$ & Yes & $27 \%$ & $1 \%$ & $1 \%$ & $36 \%$ & $3 \%$ \\
\hline $\begin{array}{l}\text { Tenderness th8/ } \\
9 \text { paraspinal } \\
\text { posterior }\end{array}$ & Yes & $16 \%$ & $1 \%$ & $0 \%$ & $22 \%$ & $3 \%$ \\
\hline $\begin{array}{l}\text { Posterior } \\
\text { vertebral } \\
\text { springing th1/2 }\end{array}$ & Yes & $7 \%$ & $4 \%$ & $0 \%$ & $5 \%$ & $12 \%$ \\
\hline $\begin{array}{l}\text { Posterior } \\
\text { vertebral } \\
\text { springing th2/3 }\end{array}$ & Yes & $12 \%$ & $29 \%$ & $1 \%$ & $14 \%$ & $25 \%$ \\
\hline
\end{tabular}


Table 2 Results of the single-stage clustering of chest pain data (Continued)

\begin{tabular}{|c|c|c|c|c|c|c|}
\hline $\begin{array}{l}\text { Posterior } \\
\text { vertebral } \\
\text { springing th3/4 }\end{array}$ & Yes & $18 \%$ & $37 \%$ & $0 \%$ & $31 \%$ & $25 \%$ \\
\hline $\begin{array}{l}\text { Posterior } \\
\text { vertebral } \\
\text { springing th4/5 }\end{array}$ & Yes & $15 \%$ & $19 \%$ & $0 \%$ & $31 \%$ & $20 \%$ \\
\hline $\begin{array}{l}\text { Posterior } \\
\text { vertebral } \\
\text { springing th } 5 / 6\end{array}$ & Yes & $19 \%$ & $6 \%$ & $0 \%$ & $16 \%$ & $10 \%$ \\
\hline $\begin{array}{l}\text { Posterior } \\
\text { vertebral } \\
\text { springing th } 6 / 7\end{array}$ & Yes & $18 \%$ & $1 \%$ & $1 \%$ & $16 \%$ & $2 \%$ \\
\hline $\begin{array}{l}\text { Posterior } \\
\text { vertebral } \\
\text { springing th7/8 }\end{array}$ & Yes & $14 \%$ & $1 \%$ & $1 \%$ & $14 \%$ & $0 \%$ \\
\hline $\begin{array}{l}\text { Posterior } \\
\text { vertebral } \\
\text { springing th8/9 }\end{array}$ & Yes & $12 \%$ & $0 \%$ & $2 \%$ & $19 \%$ & $0 \%$ \\
\hline
\end{tabular}

two subgroups described by the two-stage approach approximated classic textbook descriptions of the common diagnostic criteria for musculoskeletal chest pain (cluster 1) and atypical angina chest pain (cluster 2), and suggest that our suspicion that these data contained two subgroups may have been correct. An advantage of this approach was that two fairly distinct groups emerged that could be conceptualised as representing two different conditions. In addition, these two datadriven subgroups also differed quite markedly on patient beliefs about the anatomical cause of their condition. Such an additional finding could motivate a new view on these classic descriptions.

In comparison, the five subgroups described by the single-stage approach displayed less distinct differences. However, our overall subjective impression was that these five subgroups might also be clinically recognisable, as non-specific chest pain is a very complex complaint caused by a range of life-threatening and non-life threatening conditions. A high level of complexity in symptom presentation is in good concordance with our clinical experience that chest pain episodes are often multi-dimensional experiences accompanied by strong emotional response, that are not very well described by only the commonly defined textbook categories.

The cross-tabulation of the cluster membership between the five subgroups from single-stage clustering and the two subgroups from two-stage clustering showed evidence that both approaches seemed to reflect a similar latent data structure. However, as the singlestage clustering approach models the latent data structure of the whole data and the two-stage approach initially models the latent data structure within each domain, differences in subgroup membership across these approaches are to be expected. It should be recognised that the two-stage approach is not intended to find the identical cluster structure, but is a way to explore an alternative cluster structure. It is possible that the novel approach that we have presented may, in some circumstances, not closely mimic the data structure identified with single-stage clustering, but the results may nonetheless be more clinically useful. So it is important that clinical researchers who use two-stage clustering should judge, on a dataset by dataset basis, whether single or two-stage clustering provides more clinically interpretable subgroups that have better face validity and predictive validity.

Two-stage clustering is a form of variable reduction, as the second stage clustering involves fewer variables than the first stage. There are other methods available for variable reduction, such as Principal Components Analysis and Factor Analysis [27]. Those methods seek uni-dimensionality by identifying variables of the whole dataset that are highly correlated. However, many health domains are multi-dimensional and there may be clinical merit in retaining that knowledge. Therefore, LCA within health domains is not seeking uni-dimensionality but is identifying different within-domain scoring patterns and preserving those patterns with a new synthetic domain variable. Similarly, Principal Components Analysis and Factor Analysis are 'variable-centered' in that they seek to identify highly correlated variables, whereas LCA is 'patient-centered' in that it seeks to identify people whose scoring patterns are similar. Variable reduction can also be based on content, where content experts select the variables that are the most representative for each health domain and only use those selected 
Table 3 Results of the first stage of two-stage clustering of chest pain data

\begin{tabular}{|c|c|c|c|c|c|c|}
\hline \multicolumn{7}{|l|}{ Response option } \\
\hline \multicolumn{7}{|l|}{ Domain: Demographic } \\
\hline \multicolumn{2}{|l|}{2 clusters } & Cluster1 & \multicolumn{4}{|l|}{ Cluster2 } \\
\hline \multicolumn{2}{|l|}{ Scoring pattern label } & Older & \multicolumn{4}{|l|}{ Younger } \\
\hline \multicolumn{2}{|l|}{ Cluster Size } & $51 \%$ & \multicolumn{4}{|l|}{$49 \%$} \\
\hline $\begin{array}{l}\text { Cluster membership } \\
\text { probability }\end{array}$ & $\begin{array}{l}\text { Median } \\
\text { (interquartile } \\
\text { range) }\end{array}$ & $90 \%$ (79\% to $96 \%)$ & \multicolumn{4}{|l|}{$93 \%$ (78 \% to $100 \%)$} \\
\hline Age (years) & Mean (SD) & $62.6(5.2)$ & \multicolumn{4}{|l|}{$45.3(6.3)$} \\
\hline Gender & Female & $43 \%$ & \multicolumn{4}{|l|}{$39 \%$} \\
\hline \multicolumn{7}{|l|}{ Domain: Previous history } \\
\hline 1 cluster & & \multicolumn{5}{|c|}{ Previous history did not discriminate subgroups in two-stage clustering } \\
\hline \multicolumn{7}{|l|}{ Domain: Psychological } \\
\hline 5 clusters & & Cluster1 & Cluster2 & Cluster3 & Cluster4 & Cluster5 \\
\hline Scoring pattern label & & $\begin{array}{l}\text { Uncertain of cause of } \\
\text { pain and fearful }\end{array}$ & $\begin{array}{l}\text { Believes cause from } \\
\text { heart }\end{array}$ & $\begin{array}{l}\text { Believes both from heart } \\
\text { and musculoskeletal }\end{array}$ & $\begin{array}{l}\text { Believes cause from } \\
\text { musculoskeletal and less } \\
\text { fearful }\end{array}$ & $\begin{array}{l}\text { Believes cause from other } \\
\text { cause and less fearful }\end{array}$ \\
\hline Cluster Size & & $44 \%$ & $23 \%$ & $16 \%$ & $11 \%$ & $6 \%$ \\
\hline $\begin{array}{l}\text { Cluster membership } \\
\text { probability }\end{array}$ & $\begin{array}{l}\text { Median } \\
\text { (interquartile } \\
\text { range) }\end{array}$ & $100 \%$ (92 \% to $100 \%)$ & 99. \% (99 \% to $100 \%)$ & $100 \%(100 \%$ to $100 \%)$ & $95 \%$ (95 \% to $96 \%)$ & $77 \%$ (77 \% to $100 \%)$ \\
\hline \multirow{3}{*}{$\begin{array}{l}\text { Self-perceived 'Pain from } \\
\text { muscle or joints' }\end{array}$} & No & $10 \%$ & $100 \%$ & $0 \%$ & $0 \%$ & $75 \%$ \\
\hline & Possibly & $85 \%$ & $0 \%$ & $1 \%$ & $13 \%$ & $25 \%$ \\
\hline & Yes & $5 \%$ & $0 \%$ & $99 \%$ & $87 \%$ & $0 \%$ \\
\hline \multirow{3}{*}{$\begin{array}{l}\text { Self-perceived 'Pain from } \\
\text { heart' }\end{array}$} & No & $15 \%$ & $0 \%$ & $0 \%$ & $99 \%$ & $94 \%$ \\
\hline & Possibly & $83 \%$ & $1 \%$ & $1 \%$ & $1 \%$ & $6 \%$ \\
\hline & Yes & $3 \%$ & $99 \%$ & $99 \%$ & $0 \%$ & $0 \%$ \\
\hline Self-perceived 'Afraid' & Yes & $99 \%$ & $71 \%$ & $83 \%$ & $56 \%$ & $50 \%$ \\
\hline \multicolumn{7}{|l|}{ Domain: Pain } \\
\hline \multicolumn{2}{|l|}{2 clusters } & Cluster1 & \multicolumn{4}{|l|}{ Cluster2 } \\
\hline \multicolumn{2}{|l|}{ Scoring pattern label } & Crushing pain & \multicolumn{4}{|l|}{ Not crushing pain } \\
\hline \multicolumn{2}{|l|}{ Cluster Size } & $77 \%$ & \multicolumn{4}{|l|}{$23 \%$} \\
\hline $\begin{array}{l}\text { Cluster membership } \\
\text { probability, median } \\
\text { (interquartile range) }\end{array}$ & & $97 \%$ (94\% to $97 \%)$ & \multicolumn{4}{|l|}{$99 \%$ (96 \% to $99 \%)$} \\
\hline
\end{tabular}


Table 3 Results of the first stage of two-stage clustering of chest pain data (Continued)

\begin{tabular}{|c|c|c|c|c|}
\hline \multirow[t]{4}{*}{ Episode duration } & $<10 \min$ & $59 \%$ & $50 \%$ & \\
\hline & 10 min-1 hour & $20 \%$ & $20 \%$ & \\
\hline & $>1$ hour & $5 \%$ & $7 \%$ & \\
\hline & Continuous & $16 \%$ & $24 \%$ & \\
\hline $\begin{array}{l}\text { Pain description 'crushing } \\
\text { pain' }\end{array}$ & Yes & $100 \%$ & $25 \%$ & \\
\hline $\begin{array}{l}\text { Pain description } \\
\text { 'tenderness' }\end{array}$ & Yes & $3 \%$ & $3 \%$ & \\
\hline $\begin{array}{l}\text { Pain description 'sharp } \\
\text { pains' }\end{array}$ & Yes & $14 \%$ & $52 \%$ & \\
\hline $\begin{array}{l}\text { Pain description 'well } \\
\text { defined' }\end{array}$ & Yes & $3 \%$ & $6 \%$ & \\
\hline Pain description 'diffuse' & Yes & $6 \%$ & $5 \%$ & \\
\hline Pain description 'burning' & Yes & $4 \%$ & $8 \%$ & \\
\hline Pain description 'tingling' & Yes & $8 \%$ & $19 \%$ & \\
\hline \multicolumn{5}{|l|}{ Domain: Activity limitation } \\
\hline 3 clusters & & Cluster1 & Cluster2 & Cluster3 \\
\hline Scoring pattern label & & $\begin{array}{l}\text { None or light activity } \\
\text { limitation }\end{array}$ & $\begin{array}{l}\text { Moderate activity } \\
\text { limitation }\end{array}$ & Severe activity limitation \\
\hline Cluster Size & & $82 \%$ & $11 \%$ & $8 \%$ \\
\hline $\begin{array}{l}\text { Cluster membership } \\
\text { probability, median } \\
\text { (interquartile range) }\end{array}$ & & $\begin{array}{l}100 \%(100 \% \text { to } \\
100 \%)\end{array}$ & $86 \%$ (86 \% to $86 \%)$ & $99 \%$ (82 \% to $100 \%)$ \\
\hline $\begin{array}{l}\text { SF36 physical function sum- } \\
\text { score }\end{array}$ & Mean (SD) & $71.2(7.4)$ & $49.0(4.6)$ & $27.1(6.9)$ \\
\hline \multicolumn{5}{|c|}{ Domain: Diagnostic classification } \\
\hline 3 clusters & & Cluster1 & Cluster2 & Cluster3 \\
\hline Scoring pattern label & & Not heart & Typical angina & Maybe angina \\
\hline Cluster Size & & $37 \%$ & $35 \%$ & $28 \%$ \\
\hline $\begin{array}{l}\text { Cluster membership } \\
\text { probability }\end{array}$ & $\begin{array}{l}\text { Median } \\
\text { (interquartile } \\
\text { range) }\end{array}$ & $\begin{array}{l}100 \%(94 \% \text { to } \\
100 \%)\end{array}$ & $100 \%$ (85\% to $100 \%)$ & $97 \%$ (81 \% to $100 \%)$ \\
\hline $\begin{array}{l}\text { Danish Cardiologist's } \\
\text { Society }\end{array}$ & No angina & $64 \%$ & $3 \%$ & $2 \%$ \\
\hline
\end{tabular}

\section{Domain: Activity limitation}


Table 3 Results of the first stage of two-stage clustering of chest pain data (Continued)

\begin{tabular}{|c|c|c|c|c|c|c|c|}
\hline \multirow[t]{4}{*}{ classification 'angina' } & Typical & $0 \%$ & $89 \%$ & \multicolumn{4}{|l|}{$4 \%$} \\
\hline & Atypical & $1 \%$ & $8 \%$ & \multicolumn{4}{|l|}{$65 \%$} \\
\hline & Uncharacteri-stic & $34 \%$ & $0 \%$ & \multicolumn{4}{|l|}{$29 \%$} \\
\hline & Unstable & $0 \%$ & $1 \%$ & \multicolumn{4}{|l|}{$0 \%$} \\
\hline $\begin{array}{l}\text { Canadian Cardiovascular } \\
\text { Society }\end{array}$ & Grade 0 & $87 \%$ & $0 \%$ & \multicolumn{4}{|l|}{$8 \%$} \\
\hline \multirow[t]{3}{*}{ classification } & Grade 1 & $13 \%$ & $17 \%$ & \multicolumn{4}{|l|}{$84 \%$} \\
\hline & Grade 2 & $0 \%$ & $76 \%$ & \multicolumn{4}{|l|}{$8 \%$} \\
\hline & Grade 3 & $0 \%$ & $7 \%$ & \multicolumn{4}{|l|}{$0 \%$} \\
\hline \multicolumn{8}{|l|}{ Domain: Palpation } \\
\hline 6 clusters & & Cluster1 & Cluster2 & Cluster3 & Cluster4 & Cluster5 & Cluster6 \\
\hline Scoring pattern label & & No palpation findings & $\begin{array}{l}\text { Local } 5 / 6 \text { signs and } \\
\text { intercostal tenderness }\end{array}$ & $\begin{array}{l}\text { Local 2/3/4 signs and } \\
\text { pectoral tenderness }\end{array}$ & Pectoral tenderness & $\begin{array}{l}\text { Paraspinal and pectoral } \\
\text { tenderness }\end{array}$ & $\begin{array}{l}\text { Generalised } \\
\text { tenderness }\end{array}$ \\
\hline Cluster Size & & $22 \%$ & $19 \%$ & $18 \%$ & $16 \%$ & $16 \%$ & $8 \%$ \\
\hline $\begin{array}{l}\text { Cluster membership } \\
\text { probability, median } \\
\text { (interquartile range) }\end{array}$ & & $\begin{array}{l}100 \%(100 \% \text { to } \\
100 \%)\end{array}$ & $\begin{array}{l}100 \%(100 \% \text { to } \\
100 \%)\end{array}$ & $100 \%(100 \%$ to $100 \%)$ & $100 \%(100 \%$ to $100 \%)$ & $100 \%(100 \%$ to $100 \%)$ & $\begin{array}{l}100 \%(100 \% \\
\text { to } 100 \%)\end{array}$ \\
\hline $\begin{array}{l}\text { Sternoxiphoid springing } \\
\text { tenderness }\end{array}$ & Yes & $10 \%$ & $22 \%$ & $1 \%$ & $1 \%$ & $10 \%$ & $34 \%$ \\
\hline $\begin{array}{l}\text { Costosternal tenderness } 2 / 3 \\
\text { right }\end{array}$ & Yes & $0 \%$ & $5 \%$ & $1 \%$ & $4 \%$ & $16 \%$ & $52 \%$ \\
\hline $\begin{array}{l}\text { Costosternal tenderness } 2 / 3 \\
\text { left }\end{array}$ & Yes & $1 \%$ & $7 \%$ & $0 \%$ & $2 \%$ & $19 \%$ & $56 \%$ \\
\hline $\begin{array}{l}\text { Costosternal tenderness 3/4 } \\
\text { right }\end{array}$ & Yes & $2 \%$ & $13 \%$ & $1 \%$ & $3 \%$ & $12 \%$ & $52 \%$ \\
\hline $\begin{array}{l}\text { Costosternal tenderness } 3 / 4 \\
\text { left }\end{array}$ & Yes & $2 \%$ & $25 \%$ & $0 \%$ & $0 \%$ & $18 \%$ & $54 \%$ \\
\hline $\begin{array}{l}\text { Costosternal tenderness } 4 / 5 \\
\text { right }\end{array}$ & Yes & $1 \%$ & $28 \%$ & $1 \%$ & $1 \%$ & $4 \%$ & $51 \%$ \\
\hline $\begin{array}{l}\text { Costosternal tenderness } 4 / 5 \\
\text { left }\end{array}$ & Yes & $2 \%$ & $77 \%$ & $1 \%$ & $1 \%$ & $10 \%$ & $52 \%$ \\
\hline $\begin{array}{l}\text { Costosternal tenderness } 5 / 6 \\
\text { right }\end{array}$ & Yes & $1 \%$ & $27 \%$ & $1 \%$ & $1 \%$ & $0 \%$ & $51 \%$ \\
\hline $\begin{array}{l}\text { Costosternal tenderness } 5 / 6 \\
\text { left }\end{array}$ & Yes & $3 \%$ & $55 \%$ & $0 \%$ & $0 \%$ & $6 \%$ & $51 \%$ \\
\hline $\begin{array}{l}\text { sternoxiphoid springing } \\
\text { pain }\end{array}$ & Yes & $4 \%$ & $3 \%$ & $0 \%$ & $0 \%$ & $4 \%$ & $4 \%$ \\
\hline $\begin{array}{l}\text { Costosternal springing pain } \\
2 / 3 \text { right }\end{array}$ & Yes & $0 \%$ & $0 \%$ & $0 \%$ & $4 \%$ & $1 \%$ & $8 \%$ \\
\hline
\end{tabular}


Table 3 Results of the first stage of two-stage clustering of chest pain data (Continued)

\begin{tabular}{|c|c|c|c|c|c|c|c|}
\hline $\begin{array}{l}\text { Costosternal springing pain } \\
2 / 3 \text { left }\end{array}$ & Yes & $0 \%$ & $1 \%$ & $0 \%$ & $1 \%$ & $4 \%$ & $10 \%$ \\
\hline $\begin{array}{l}\text { Costosternal springing pain } \\
3 / 4 \text { right }\end{array}$ & Yes & $0 \%$ & $2 \%$ & $1 \%$ & $2 \%$ & $2 \%$ & $10 \%$ \\
\hline $\begin{array}{l}\text { Costosternal springing pain } \\
3 / 4 \text { left }\end{array}$ & Yes & $1 \%$ & $7 \%$ & $0 \%$ & $0 \%$ & $5 \%$ & $10 \%$ \\
\hline $\begin{array}{l}\text { Costosternal springing pain } \\
4 / 5 \text { right }\end{array}$ & Yes & $0 \%$ & $8 \%$ & $1 \%$ & $0 \%$ & $1 \%$ & $6 \%$ \\
\hline $\begin{array}{l}\text { Costosternal springing pain } \\
4 / 5 \text { left }\end{array}$ & Yes & $1 \%$ & $36 \%$ & $0 \%$ & $0 \%$ & $0 \%$ & $4 \%$ \\
\hline $\begin{array}{l}\text { Costosternal springing pain } \\
5 / 6 \text { right }\end{array}$ & Yes & $0 \%$ & $9 \%$ & $0 \%$ & $0 \%$ & $0 \%$ & $4 \%$ \\
\hline $\begin{array}{l}\text { Costosternal springing pain } \\
5 / 6 \text { left }\end{array}$ & Yes & $0 \%$ & $29 \%$ & $0 \%$ & $1 \%$ & $2 \%$ & $6 \%$ \\
\hline \multirow{2}{*}{$\begin{array}{l}\text { Muscle tenderness } \\
\text { pectoralis major left }\end{array}$} & Tenderness & $1 \%$ & $19 \%$ & $78 \%$ & $62 \%$ & $38 \%$ & $54 \%$ \\
\hline & Pain & $0 \%$ & $3 \%$ & $20 \%$ & $28 \%$ & $4 \%$ & $24 \%$ \\
\hline \multirow{2}{*}{$\begin{array}{l}\text { Muscle tenderness } \\
\text { pectoralis minor left }\end{array}$} & Tenderness & $2 \%$ & $31 \%$ & $83 \%$ & $75 \%$ & $40 \%$ & $66 \%$ \\
\hline & Pain & $0 \%$ & $6 \%$ & $13 \%$ & $8 \%$ & $4 \%$ & $20 \%$ \\
\hline \multirow{2}{*}{$\begin{array}{l}\text { Muscle tenderness } \\
\text { intercostal } 2 / 3 \text { left }\end{array}$} & Tenderness & $1 \%$ & $6 \%$ & $58 \%$ & $42 \%$ & $24 \%$ & $84 \%$ \\
\hline & Pain & $1 \%$ & $0 \%$ & $3 \%$ & $1 \%$ & $0 \%$ & $12 \%$ \\
\hline \multirow{2}{*}{$\begin{array}{l}\text { Muscle tenderness } \\
\text { intercostal } 3 / 4 \text { left }\end{array}$} & Tenderness & $1 \%$ & $20 \%$ & $36 \%$ & $20 \%$ & $18 \%$ & $90 \%$ \\
\hline & Pain & $1 \%$ & $2 \%$ & $0 \%$ & $0 \%$ & $1 \%$ & $8 \%$ \\
\hline \multirow{2}{*}{$\begin{array}{l}\text { Muscle tenderness } \\
\text { intercostal } 4 / 5 \text { left }\end{array}$} & Tenderness & $3 \%$ & $46 \%$ & $12 \%$ & $10 \%$ & $5 \%$ & $91 \%$ \\
\hline & Pain & $1 \%$ & $15 \%$ & $0 \%$ & $1 \%$ & $1 \%$ & $6 \%$ \\
\hline \multirow{2}{*}{$\begin{array}{l}\text { Muscle tenderness } \\
\text { intercostal 5/6 left }\end{array}$} & Tenderness & $2 \%$ & $57 \%$ & $5 \%$ & $3 \%$ & $4 \%$ & $89 \%$ \\
\hline & Pain & $0 \%$ & $21 \%$ & $0 \%$ & $0 \%$ & $0 \%$ & $6 \%$ \\
\hline \multirow{2}{*}{$\begin{array}{l}\text { Muscle tenderness } \\
\text { intercostal } 6 / 7 \text { left }\end{array}$} & Tenderness & $1 \%$ & $42 \%$ & $1 \%$ & $1 \%$ & $0 \%$ & $82 \%$ \\
\hline & Pain & $0 \%$ & $14 \%$ & $0 \%$ & $1 \%$ & $1 \%$ & $8 \%$ \\
\hline \multirow{2}{*}{$\begin{array}{l}\text { Muscle tenderness } \\
\text { pectoralis major right }\end{array}$} & Tenderness & $0 \%$ & $16 \%$ & $75 \%$ & $74 \%$ & $28 \%$ & $56 \%$ \\
\hline & Pain & $0 \%$ & $0 \%$ & $21 \%$ & $16 \%$ & $4 \%$ & $24 \%$ \\
\hline
\end{tabular}


Table 3 Results of the first stage of two-stage clustering of chest pain data (Continued)

\begin{tabular}{|c|c|c|c|c|c|c|c|}
\hline \multirow{2}{*}{$\begin{array}{l}\text { Muscle tenderness } \\
\text { pectoralis minor right }\end{array}$} & Tenderness & $1 \%$ & $17 \%$ & $80 \%$ & $68 \%$ & $33 \%$ & $66 \%$ \\
\hline & Pain & $0 \%$ & $0 \%$ & $11 \%$ & $7 \%$ & $1 \%$ & $20 \%$ \\
\hline \multirow{2}{*}{$\begin{array}{l}\text { Muscle tenderness } \\
\text { intercostal } 2 / 3 \text { right }\end{array}$} & Tenderness & $0 \%$ & $9 \%$ & $59 \%$ & $38 \%$ & $16 \%$ & $90 \%$ \\
\hline & Pain & $0 \%$ & $1 \%$ & $0 \%$ & $1 \%$ & $1 \%$ & $8 \%$ \\
\hline \multirow{2}{*}{$\begin{array}{l}\text { Muscle tenderness } \\
\text { intercostal } 3 / 4 \text { right }\end{array}$} & Tenderness & $1 \%$ & $15 \%$ & $32 \%$ & $15 \%$ & $9 \%$ & $90 \%$ \\
\hline & Pain & $0 \%$ & $0 \%$ & $0 \%$ & $1 \%$ & $1 \%$ & $6 \%$ \\
\hline \multirow{2}{*}{$\begin{array}{l}\text { Muscle tenderness } \\
\text { intercostal } 4 / 5 \text { right }\end{array}$} & Tenderness & $0 \%$ & $27 \%$ & $10 \%$ & $4 \%$ & $4 \%$ & $90 \%$ \\
\hline & Pain & $0 \%$ & $2 \%$ & $0 \%$ & $0 \%$ & $0 \%$ & $4 \%$ \\
\hline \multirow{2}{*}{$\begin{array}{l}\text { Muscle tenderness } \\
\text { intercostal } 5 / 6 \text { right }\end{array}$} & Tenderness & $0 \%$ & $30 \%$ & $3 \%$ & $1 \%$ & $0 \%$ & $89 \%$ \\
\hline & Pain & $0 \%$ & $4 \%$ & $0 \%$ & $0 \%$ & $0 \%$ & $4 \%$ \\
\hline \multirow{2}{*}{$\begin{array}{l}\text { Muscle tenderness } \\
\text { intercostal } 6 / 7 \text { right }\end{array}$} & Tenderness & $0 \%$ & $17 \%$ & $2 \%$ & $2 \%$ & $0 \%$ & $84 \%$ \\
\hline & Pain & $0 \%$ & $5 \%$ & $0 \%$ & $0 \%$ & $0 \%$ & $4 \%$ \\
\hline $\begin{array}{l}\text { Tenderness c } 4 / 5 \text { paraspinal } \\
\text { posterior }\end{array}$ & Yes & $3 \%$ & $6 \%$ & $6 \%$ & $1 \%$ & $17 \%$ & $27 \%$ \\
\hline $\begin{array}{l}\text { Tenderness c } 5 / 6 \text { paraspinal } \\
\text { posterior }\end{array}$ & Yes & $4 \%$ & $13 \%$ & $11 \%$ & $3 \%$ & $20 \%$ & $26 \%$ \\
\hline $\begin{array}{l}\text { Tenderness c } 6 / 7 \text { paraspinal } \\
\text { posterior }\end{array}$ & Yes & $3 \%$ & $13 \%$ & $8 \%$ & $3 \%$ & $12 \%$ & $19 \%$ \\
\hline $\begin{array}{l}\text { Tenderness } c 7 / \text { th } 1 \\
\text { paraspinal posterior }\end{array}$ & Yes & $1 \%$ & $8 \%$ & $18 \%$ & $0 \%$ & $9 \%$ & $21 \%$ \\
\hline $\begin{array}{l}\text { Tenderness th } 1 / 2 \\
\text { paraspinal posterior }\end{array}$ & Yes & $2 \%$ & $14 \%$ & $50 \%$ & $0 \%$ & $20 \%$ & $31 \%$ \\
\hline $\begin{array}{l}\text { Tenderness th } 2 / 3 \\
\text { paraspinal posterior }\end{array}$ & Yes & $3 \%$ & $27 \%$ & $89 \%$ & $0 \%$ & $46 \%$ & $51 \%$ \\
\hline $\begin{array}{l}\text { Tenderness th } 3 / 4 \\
\text { paraspinal posterior }\end{array}$ & Yes & $1 \%$ & $33 \%$ & $78 \%$ & $0 \%$ & $54 \%$ & $61 \%$ \\
\hline $\begin{array}{l}\text { Tenderness th } 4 / 5 \\
\text { paraspinal posterior }\end{array}$ & Yes & $1 \%$ & $38 \%$ & $40 \%$ & $1 \%$ & $59 \%$ & $59 \%$ \\
\hline $\begin{array}{l}\text { Tenderness th5/6 } \\
\text { paraspinal posterior }\end{array}$ & Yes & $1 \%$ & $45 \%$ & $13 \%$ & $0 \%$ & $53 \%$ & $41 \%$ \\
\hline $\begin{array}{l}\text { Tenderness th } 6 / 7 \\
\text { paraspinal posterior }\end{array}$ & Yes & $1 \%$ & $43 \%$ & $1 \%$ & $0 \%$ & $45 \%$ & $35 \%$ \\
\hline
\end{tabular}


Table 3 Results of the first stage of two-stage clustering of chest pain data (Continued)

\begin{tabular}{|c|c|c|c|c|c|c|c|}
\hline $\begin{array}{l}\text { Tenderness th7/8 } \\
\text { paraspinal posterior }\end{array}$ & Yes & $3 \%$ & $27 \%$ & $2 \%$ & $0 \%$ & $26 \%$ & $33 \%$ \\
\hline $\begin{array}{l}\text { Tenderness th8/9 } \\
\text { paraspinal posterior }\end{array}$ & Yes & $2 \%$ & $12 \%$ & $0 \%$ & $0 \%$ & $17 \%$ & $25 \%$ \\
\hline $\begin{array}{l}\text { Posterior vertebral } \\
\text { springing th } 1 / 2\end{array}$ & Yes & $0 \%$ & $8 \%$ & $10 \%$ & $2 \%$ & $4 \%$ & $2 \%$ \\
\hline $\begin{array}{l}\text { Posterior vertebral } \\
\text { springing th } 2 / 3\end{array}$ & Yes & $1 \%$ & $7 \%$ & $41 \%$ & $11 \%$ & $18 \%$ & $17 \%$ \\
\hline $\begin{array}{l}\text { Posterior vertebral } \\
\text { springing th } 3 / 4\end{array}$ & Yes & $0 \%$ & $21 \%$ & $51 \%$ & $11 \%$ & $21 \%$ & $26 \%$ \\
\hline $\begin{array}{l}\text { Posterior vertebral } \\
\text { springing th } 4 / 5\end{array}$ & Yes & $0 \%$ & $15 \%$ & $31 \%$ & $5 \%$ & $18 \%$ & $28 \%$ \\
\hline $\begin{array}{l}\text { Posterior vertebral } \\
\text { springing th } 5 / 6\end{array}$ & Yes & $0 \%$ & $14 \%$ & $12 \%$ & $0 \%$ & $24 \%$ & $17 \%$ \\
\hline $\begin{array}{l}\text { Posterior vertebral } \\
\text { springing th } 6 / 7\end{array}$ & Yes & $1 \%$ & $19 \%$ & $2 \%$ & $0 \%$ & $19 \%$ & $11 \%$ \\
\hline $\begin{array}{l}\text { Posterior vertebral } \\
\text { springing th7/8 }\end{array}$ & Yes & $2 \%$ & $12 \%$ & $1 \%$ & $2 \%$ & $14 \%$ & $11 \%$ \\
\hline $\begin{array}{l}\text { Posterior vertebral } \\
\text { springing th } 8 / 9\end{array}$ & Yes & $5 \%$ & $9 \%$ & $1 \%$ & $0 \%$ & $10 \%$ & $19 \%$ \\
\hline
\end{tabular}


Table 4 Results of the second stage of two-stage clustering (described using the prevalence of first stage cluster membership)

\begin{tabular}{|c|c|c|c|}
\hline \multirow{2}{*}{$\begin{array}{l}\text { Domain } \\
\text { Subgroup label }\end{array}$} & & \multirow{2}{*}{$\begin{array}{l}\text { Cluster } 1 \\
\text { Uncertain of cause and fearful, } \\
\text { but not heart-related - with local } \\
\text { thoracic } 5 / 6 \text { palpation findings. }\end{array}$} & \multirow{2}{*}{$\begin{array}{l}\text { Cluster } 2 \\
\text { Believes pain to be heart- or } \\
\text { musculoskelet-al-related with crushing } \\
\text { pain, and local thoracic } 2 / 3 \text { signs and } \\
\text { pectoral tenderness. }\end{array}$} \\
\hline & & & \\
\hline Cluster size & & $51 \%$ & $49 \%$ \\
\hline $\begin{array}{l}\text { Cluster membership } \\
\text { probability }\end{array}$ & Median (interquartile range) & $100 \%$ (99 \% to $100 \%)$ & $100 \%$ (98 \% to $100 \%)$ \\
\hline \multirow[t]{2}{*}{ Demographic } & Younger & $42 \% *$ & $62 \%$ \\
\hline & Older & $58 \%$ & $38 \%$ \\
\hline \multirow[t]{2}{*}{ Previous history } & & $0 \%$ & $0 \%$ \\
\hline & Only one cluster & $100 \%$ & $100 \%$ \\
\hline \multirow[t]{5}{*}{ Psychological } & Uncertain of cause of pain and fearful & $88 \%$ & $2 \%$ \\
\hline & Believes cause from heart & $1 \%$ & $44 \%$ \\
\hline & Believes both from heart and musculoskeletal & $0 \%$ & $31 \%$ \\
\hline & $\begin{array}{l}\text { Believes cause from musculoskeletal and less } \\
\text { fearful }\end{array}$ & $6 \%$ & $14 \%$ \\
\hline & $\begin{array}{l}\text { Believes cause from other cause and less } \\
\text { fearful }\end{array}$ & $5 \%$ & $8 \%$ \\
\hline \multirow[t]{2}{*}{ Pain } & Crushing pain & $74 \%$ & $89 \%$ \\
\hline & Not crushing pain & $26 \%$ & $11 \%$ \\
\hline \multirow[t]{3}{*}{ Activity limitation } & None or light activity limitation & $88 \%$ & $76 \%$ \\
\hline & Moderate activity limitation & $8 \%$ & $13 \%$ \\
\hline & Severe activity limitation & $4 \%$ & $11 \%$ \\
\hline \multirow[t]{3}{*}{ Diagnosticclassification } & Not heart & $71 \%$ & $1 \%$ \\
\hline & Typical angina & $13 \%$ & $61 \%$ \\
\hline & Maybe angina & $16 \%$ & $38 \%$ \\
\hline \multirow[t]{6}{*}{ Palpation } & No palpation findings & $23 \%$ & $23 \%$ \\
\hline & Local 5/6 signs and intercostal tenderness & $36 \%$ & $1 \%$ \\
\hline & Local 2/3/4 signs and pectoral tenderness & $4 \%$ & $32 \%$ \\
\hline & Pectoral tenderness & $3 \%$ & $30 \%$ \\
\hline & Paraspinal and pectoral tenderness & $23 \%$ & $8 \%$ \\
\hline & Generalised tenderness & $11 \%$ & $6 \%$ \\
\hline
\end{tabular}

*Proportions are those of the people within each cluster on each domain (vertical proportions), as each category within a domain is mutually exclusive

variables in single-stage clustering. However, clustering approaches are often applied when there is inadequate prior knowledge about which measurements would best inform subgroup formation.

\section{Conclusions}

In conclusion, we have suggested a new approach to using statistical clustering techniques to identify clinically useful subgroups of patients. Research designs, statistical methods and outcome metrics suitable for performing that testing have also been described. While the paper illustrates this approach using a practical example of (chest pain) baseline data, due to limitations in the data, it does not extend that analysis into testing the example's clinical importance or implications for prognosis and treatment. We are undertaking such analysis on data from primary and secondary care patients with low back pain, and the results will be comprehensively reported in subsequent papers. This novel statistical approach has a number of potential benefits but requires broad testing, in multiple patient samples, to determine if it is useful. That is likely to be context-specific, depending on the characteristics of the available data and the research question being asked of it. 
Table 5 Results of the second stage of two-stage clustering (described in the measurement units of the original variables)

\begin{tabular}{|c|c|c|c|}
\hline & Response option & Cluster1 & Cluster2 \\
\hline Subgroup label & & $\begin{array}{l}\text { Uncertain of cause and fearful, } \\
\text { but not heart-related - with local } \\
\text { thoracic } 5 / 6 \text { palpation findings. }\end{array}$ & $\begin{array}{l}\text { Believes pain to be heart- or } \\
\text { musculoskelet-al-related with } \\
\text { crushing pain, and local thoracic } \\
2 / 3 \text { signs and pectoral tendernes }\end{array}$ \\
\hline
\end{tabular}

Demographic domain

Age

Gender

Previous history domain

Cardiovascular risk factor Index

Psychological domain

Self-perceived 'Pain from muscle or joints'

Self-perceived 'Pain from heart'

Self-perceived 'Afraid'

Pain domain

Episode duration

Pain description 'crushing pain'

Pain description 'tenderness'

Pain description 'sharp pains'

Pain description 'well defined'

Pain description 'diffuse'

Pain description 'burning'

Pain description 'tingling'

Activity limitation domain

SF36 physical function sum-score

Diagnostic classification domain

Danish Cardiologist's Society

classification 'angina'

Canadian Cardiovascular Society

classification

Palpation domain

Sternoxiphoid springing tend

Costosternal springing tenderness $2 / 3$ right

Costosternal springing tenderness $2 / 3$ left

Costosternal springing tenderness 3/4 right

Costosternal springing tenderness $3 / 4$ left
Mean (SD)

Female

Mean (SD)

Possibly

Yes

Possibly

Yes

Yes

$52.2(11.0)$

$44 \%$

56.5 (9.2)

$39 \%$

$22.2(1.1)$

$1.9(1.1)$

$79 \%$

$1 \%$

$46 \%$

$1 \%$

$76 \%$

$71 \%$

$<10 \mathrm{~min}$

$34 \%$

$57 \%$

10 min-1 hour

$24 \%$

$>1$ hour

$5 \%$

Continuous

Yes

$37 \%$

$76 \%$

$0 \%$

$25 \%$

$3 \%$

$2 \%$

$6 \%$

$7 \%$

67.8 (SD 13.4)

61.5 (SD 16.1)

No angina

$49 \%$

Typical

$9 \%$

Atypical

$14 \%$

Uncharacteristic

$28 \%$

Unstable

$0 \%$

Grade 0

Grade 1

$67 \%$

$21 \%$

$11 \%$

$0 \%$

Grade 3

Yes

Yes

Yes

Yes

Yes
$0 \%$

$56 \%$

$30 \%$

$14 \%$

$20 \%$

$6 \%$

$17 \%$

$90 \%$

$6 \%$

$20 \%$

$4 \%$

$8 \%$

$5 \%$

$13 \%$

$0 \%$

$1 \%$

$47 \%$

$46 \%$

$5 \%$

$2 \%$

$4 \%$

$4 \%$

$3 \%$

$3 \%$ 
Table 5 Results of the second stage of two-stage clustering (described in the measurement units of the original variables) (Continued)

\begin{tabular}{|c|c|c|c|}
\hline Costosternal springing tenderness $4 / 5$ right & Yes & $20 \%$ & $1 \%$ \\
\hline Costosternal springing tenderness $4 / 5$ left & Yes & $40 \%$ & $2 \%$ \\
\hline Costosternal springing tenderness $5 / 6$ right & Yes & $18 \%$ & $1 \%$ \\
\hline Costosternal springing tenderness $5 / 6$ left & Yes & $32 \%$ & $1 \%$ \\
\hline sternoxiphoid springing pain & Yes & $5 \%$ & $0 \%$ \\
\hline Costosternal springing pain $2 / 3$ right & Yes & $2 \%$ & $1 \%$ \\
\hline Costosternal springing pain 2/3 left & Yes & $3 \%$ & $1 \%$ \\
\hline Costosternal springing pain 3/4 right & Yes & $3 \%$ & $1 \%$ \\
\hline Costosternal springing pain 3/4 left & Yes & $6 \%$ & $1 \%$ \\
\hline Costosternal springing pain $4 / 5$ right & Yes & $5 \%$ & $0 \%$ \\
\hline Costosternal springing pain $4 / 5$ left & Yes & $15 \%$ & $0 \%$ \\
\hline Costosternal springing pain 5/6 right & Yes & $4 \%$ & $0 \%$ \\
\hline Costosternal springing pain 5/6 left & Yes & $13 \%$ & $0 \%$ \\
\hline Muscle tenderness pectoralis major left & Tenderness & $23 \%$ & $54 \%$ \\
\hline Costosternal springing pain 5/6 left & Pain & $6 \%$ & $17 \%$ \\
\hline \multirow[t]{2}{*}{ Muscle tenderness pectoralis minor left } & Tenderness & $31 \%$ & $60 \%$ \\
\hline & Pain & $7 \%$ & $7 \%$ \\
\hline \multirow[t]{2}{*}{ Muscle tenderness intercostal 2/3 left } & Tenderness & $18 \%$ & $41 \%$ \\
\hline & Pain & $1 \%$ & $3 \%$ \\
\hline \multirow[t]{2}{*}{ Muscle tenderness intercostal 3/4 left } & Tenderness & $23 \%$ & $25 \%$ \\
\hline & Pain & $2 \%$ & $1 \%$ \\
\hline \multirow[t]{2}{*}{ Muscle tenderness intercostal 4/5 left } & Tenderness & $29 \%$ & $14 \%$ \\
\hline & Pain & $7 \%$ & $1 \%$ \\
\hline \multirow[t]{2}{*}{ Muscle tenderness intercostal 5/6 left } & Tenderness & $32 \%$ & $9 \%$ \\
\hline & Pain & $9 \%$ & $0 \%$ \\
\hline \multirow[t]{2}{*}{ Muscle tenderness intercostal $6 / 7$ left } & Tenderness & $21 \%$ & $7 \%$ \\
\hline & Pain & $7 \%$ & $0 \%$ \\
\hline \multirow[t]{2}{*}{ Muscle tenderness pectoralis major right } & Tenderness & $21 \%$ & $55 \%$ \\
\hline & Pain & $3 \%$ & $15 \%$ \\
\hline \multirow[t]{2}{*}{ Muscle tenderness pectoralis minor right } & Tenderness & $25 \%$ & $54 \%$ \\
\hline & Pain & $2 \%$ & $8 \%$ \\
\hline \multirow[t]{2}{*}{ Muscle tenderness intercostal 2/3 right } & Tenderness & $18 \%$ & $39 \%$ \\
\hline & Pain & $2 \%$ & $1 \%$ \\
\hline \multirow[t]{2}{*}{ Muscle tenderness intercostal 3/4 right } & Tenderness & $17 \%$ & $23 \%$ \\
\hline & Pain & $1 \%$ & $0 \%$ \\
\hline \multirow[t]{2}{*}{ Muscle tenderness intercostal $4 / 5$ right } & Tenderness & $20 \%$ & $11 \%$ \\
\hline & Pain & $1 \%$ & $0 \%$ \\
\hline \multirow[t]{2}{*}{ Muscle tenderness intercostal 5/6 right } & Tenderness & $21 \%$ & $7 \%$ \\
\hline & Pain & $2 \%$ & $0 \%$ \\
\hline \multirow[t]{2}{*}{ Muscle tenderness intercostal $6 / 7$ right } & Tenderness & $16 \%$ & $6 \%$ \\
\hline & Pain & $2 \%$ & $0 \%$ \\
\hline Tenderness $c 4 / 5$ paraspinal posterior & Yes & $11 \%$ & $5 \%$ \\
\hline Tenderness c $5 / 6$ paraspinal posterior & Yes & $16 \%$ & $6 \%$ \\
\hline Tenderness c6/7 paraspinal posterior & Yes & $12 \%$ & $5 \%$ \\
\hline
\end{tabular}


Table 5 Results of the second stage of two-stage clustering (described in the measurement units of the original variables) (Continued)

\begin{tabular}{llll}
\hline Tenderness c7/th1 paraspinal posterior & Yes & $7 \%$ & $10 \%$ \\
Tenderness th1/2 paraspinal posterior & Yes & $14 \%$ & $22 \%$ \\
Tenderness th2/3 paraspinal posterior & Yes & $27 \%$ & $40 \%$ \\
Tenderness th3/4 paraspinal posterior & Yes & $32 \%$ & $36 \%$ \\
Tenderness th4/5 paraspinal posterior & Yes & $35 \%$ & $23 \%$ \\
Tenderness th5/6 paraspinal posterior & Yes & $35 \%$ & $11 \%$ \\
Tenderness th6/7 paraspinal posterior & Yes & $31 \%$ & $6 \%$ \\
Tenderness th7/8 paraspinal posterior & Yes & $22 \%$ & $4 \%$ \\
Tenderness th8/9 paraspinal posterior & Yes & $16 \%$ & $1 \%$ \\
Posterior vertebral springing th1/2 & Yes & $6 \%$ & $4 \%$ \\
Posterior vertebral springing th2/3 & Yes & $9 \%$ & $21 \%$ \\
Posterior vertebral springing th3/4 & Yes & $16 \%$ & $25 \%$ \\
Posterior vertebral springing th4/5 & Yes & $14 \%$ & $15 \%$ \\
Posterior vertebral springing th5/6 & Yes & $14 \%$ & $7 \%$ \\
Posterior vertebral springing th6/7 & Yes & $14 \%$ & $3 \%$ \\
Posterior vertebral springing th7/8 & Yes & $10 \%$ & $11 \%$ \\
Posterior vertebral springing th8/9 & Yes & & $3 \%$
\end{tabular}

Table 6 Cross-tabulation of cluster membership

\begin{tabular}{|c|c|c|}
\hline & Two-stage clustering & \\
\hline & Subgroup 1 & Subgroup 2 \\
\hline & $\begin{array}{l}\text { Uncertain of cause and fearful, but not heart- } \\
\text { related - with local thoracic } 5 / 6 \text { palpation } \\
\text { findings. }\end{array}$ & $\begin{array}{l}\text { Believes pain to be heart- or musculoskeletal-related with } \\
\text { crushing pain, and local thoracic } 2 / 3 \text { signs and pectoral } \\
\text { tenderness. }\end{array}$ \\
\hline \multicolumn{3}{|l|}{ Single stage clustering } \\
\hline \multicolumn{3}{|l|}{ Subgroup 1} \\
\hline $\begin{array}{l}\text { Low activity limitation, high fear, } \\
\text { few palpation findings }\end{array}$ & 173 & 13 \\
\hline \multicolumn{3}{|l|}{ Subgroup 2} \\
\hline $\begin{array}{l}\text { Typical and atypical angina, short } \\
\text { episode, pectoral tenderness }\end{array}$ & 9 & 144 \\
\hline \multicolumn{3}{|l|}{ Subgroup 3} \\
\hline No palpation findings & 64 & \\
\hline \multicolumn{3}{|l|}{ Subgroup 4} \\
\hline $\begin{array}{l}\text { Female, high fear, diffuse anterior } \\
\text { tenderness }\end{array}$ & 44 & 16 \\
\hline \multicolumn{3}{|l|}{ Subgroup 5} \\
\hline $\begin{array}{l}\text { Typical angina, short episode, high } \\
\text { activity limitation, pectoral } \\
\text { tenderness }\end{array}$ & 5 & 35 \\
\hline
\end{tabular}




\section{Competing interests}

The authors declare that they have no financial or non-financial competing interests.

\section{Authors' contributions}

The concept of the paper originated from PK. All authors were involved in the design of the study, the drafting and revision of the manuscript, and gave final approval of the manuscript.

\section{Acknowledgements}

PK is partially funded, and AK, MS and HWC fully funded, by the Danish Fund for Chiropractic Research and Post-graduate Education. No funding source played any role in the scientific conduct of the study.

\section{Author details}

'Department of Sports Science and Clinical Biomechanics, University of Southern Denmark, Campusvej 55, Odense M 5230, Denmark. ${ }^{2}$ Nordic Institute of Chiropractic and Clinical Biomechanics, Odense, Denmark.

Received: 17 December 2014 Accepted: 9 June 2015

Published online: 02 July 2015

\section{References}

1. Hingorani AD, Windt DA, Riley RD, Abrams K, Moons KG, Steyerberg EW, et al. Prognosis research strategy (PROGRESS) 4: Stratified medicine research. BMJ. 2013;346, e5793.

2. Kroenke K, Harris L. Symptoms research: a fertile field. Ann Intern Med. 2001;134(9 Pt 2):801-2

3. Amirall D, Compton SN, Gunlicks-Stoessel M, Duan N, Murphy S. Designing a pilot sequential multiple assignment randomized trial for developing an adaptive treatment strategy. Stat Med. 2012;31:1887-902.

4. Von Korff KM, Moore JC: Stepped care for back pain: activating approaches for primary care. Ann Intern Med 2001;134(9 Pt 2):911-7.

5. Hayden JA, Côté P, Steenstra IA, Bombardier C. Group ftQ-LW: Identifying phases of investigation helps planning, appraising and applying the results of explanatory prognosis studies. J Clin Epidemiol. 2008:61(6):552-60.

6. Kotsiantis S, Pintelas P: Recent Advances in Clustering: A Brief Survey, WSEAS Transactions on Information Science and Applications 2004;1(1):73-81.

7. von Eye A, Bogat GA, Rhodes JE. Variable-oriented and person-oriented perspectives of analysis: the example of alcohol consumption in adolescence. J Adolesc. 2006;29(6):981-1004.

8. Schellingerhout JM, Heymans MW, Verhagen AP, Lewis M, de Vet HC, Koes BW. Prognosis of patients with nonspecific neck pain: development and external validation of a prediction rule for persistence of complaints. Spine (Phila Pa 1976). 2010;35(17):E827-835.

9. Kent $P$, Keating $J$, Leboeuf-Yde $C$ : Research methods for subgrouping low back pain. BMC Research Methods 2010, 10:62doi:10.1186/1471-2288-10-62.

10. Beneciuk JM, Robinson ME, George SZ. Low back pain subgroups using fear-avoidance model measures: results of a cluster analysis. Clin J Pain. 2012;28(8):658-66

11. Westman AE, Boersma K, Leppert J, Linton SJ. Fear-avoidance beliefs, catastrophizing, and distress: a longitudinal subgroup analysis on patients with musculoskeletal pain. Clin J Pain. 2011;27(7):567-77.

12. Hill JC, Dunn KM, Lewis M, Mullis R, Main C. J., Foster NE, Hay EM: A primary care back pain screening tool: Identifying patient subgroups for initial treatment. Arthritis Rheum. 2008:59(5):632-41.

13. Jorgensen CK, Fink P, Olesen F. Psychological distress and somatisation as prognostic factors in patients with musculoskeletal illness in general practice. Br J Gen Pract. 2000;50(456):537-41.

14. Pincus T, Burton A, Vogel S, Field A. A systematic review of psychological factors as predictors of chronicity/disability in prospective cohorts of low back pain. Spine. 2002;27(5):E109-20.

15. Tetko IV, Livingstone DJ, Luik Al. Neural network studies. 1. Comparison of overfitting and overtraining. J Chem Inf Comput Sci. 1995;35(5):826-33.

16. Collins LM, Lanza ST. Latent class and latent transition analysis for the social, behavioral, and health sciences. New York: Wiley; 2010.

17. Beneciuk JM, Robinson ME, George SZ: Subgrouping For Patients With Low Back Pain: A Multidimensional Approach Incorporating Cluster Analysis \& The STarT Back Screening Tool. J Pain 2015;16(1):19-30. doi:10.1016/ j.jpain.2014.10.004
18. Foster NE, Hill JC, Hay EM. Subgrouping patients with low back pain in primary care: are we getting any better at it? Man Ther. 2011;16(1):3-8.

19. Fritz JM, Cleland JA, Childs JD. Subgrouping patients with low back pain: evolution of a classification approach to physical therapy. J Orthop Sports Phys Ther. 2007:37(6):290-302.

20. World Health Organisation. International Classification of Functioning, Disability and Health. Geneva, Switzerland: World Health Organisation; 2001.

21. Takahashi O, Cook EF, Nakamura T, Saito J, Ikawa F, Fukui T. Risk stratification for in-hospital mortality in spontaneous intracerebral haemorrhage: a Classification and Regression Tree analysis. QJM. 2006;99(11):743-50.

22. McGinn TG, Guyatt GH, Wyer PC, Naylor CD, Stiell IG, Richardson WS. Users' guides to the medical literature: XXII: how to use articles about clinical decision rules. JAMA. 2000:284:79-84.

23. Stochkendahl MJ, Christensen HW, Vach W, Hoilund-Carlsen PF, Haghfelt T, Hartvigsen J. Diagnosis and treatment of musculoskeletal chest pain: design of a multi-purpose trial. BMC Musculoskelet Disord. 2008;9:40.

24. Stochkendahl MJ, Christensen HW, Vach W, Hoilund-Carlsen PF, Haghfelt T, Hartvigsen J. Chiropractic treatment vs self-management in patients with acute chest pain: a randomized controlled trial of patients without acute coronary syndrome. J Manipulative Physiol Ther. 2012;35(1):7-17.

25. Christensen HW, Vach W, Gichangi A, Manniche C, Haghfelt T, Hoilund-Carlsen PF. Manual therapy for patients with stable angina pectoris: a nonrandomized open prospective trial. J Manipulative Physiol Ther. 2005:28(9):654-61

26. Christensen HW, Vach W, Gichangi A, Manniche C, Haghfelt T, Hoilund Carlsen PF. Cervicothoracic angina identified by case history and palpation findings in patients with stable angina pectoris. J Manipulative Physiol Ther 2005:28(5):303-11.

27. Vigneau E, Sahmer K, Qannariand EM, Bertrand D. Clustering of variables to analyze spectral data. J Chemometrics. 2005;19:122-8.

\section{Submit your next manuscript to BioMed Central and take full advantage of:}

- Convenient online submission

- Thorough peer review

- No space constraints or color figure charges

- Immediate publication on acceptance

- Inclusion in PubMed, CAS, Scopus and Google Scholar

- Research which is freely available for redistribution 